\title{
Stabilization of discrete linear repetitive processes with switched dynamics
}

\author{
J. Bochniak · K. Galkowski • E. Rogers • D. Mehdi • \\ O. Bachelier • A. Kummert
}

Received: 2 February 2005 / Revised 11 November 2005 /

Accepted: 18 November 2005 / Published online: April 2006

CSpringer Science+Business Media, LLC 2006

\begin{abstract}
Repetitive processes are a distinct class of $2 D$ systems (i.e. information propagation in two independent directions) of both systems theoretic and applications interest. They cannot be controlled by direct extension of existing techniques from either standard (termed $1 D$ here) or $2 D$ systems theory. Here we give new results on the relatively open problem of the design of physically based control laws. These results are for a sub-class of discrete linear repetitive processes with switched dynamics in both independent directions of information propagation.
\end{abstract}

Keywords Repetitive processes $\cdot$ Switched dynamics $\cdot$ LMIs

\section{Introduction}

The essential unique characteristic of a repetitive process (also termed a multipass process in the early literature) can be illustrated by considering machining operations where the material or workpiece involved is processed by a series of sweeps, or passes, of the processing tool. Assuming the pass length $\alpha<+\infty$ to be constant, the output vector, or pass profile, $y_{k}(p)$, $p=0,1, \ldots,(\alpha-1),(p$ being the independent spatial or temporal variable $)$, generated on

\footnotetext{
J. Bochniak · K. Galkowski ( $\square)$

Institute of Control and Computation Engineering, University of Zielona Gora, Zielona Gora, Poland

e-mail: K.Galkowski@issi.uz.zgora.pl

E. Rogers

School of Electronics and Computer Science,

University of Southampton, Southampton, United Kingdom

D. Mehdi · O. Bachelier

LAII-ESIP, University of Poitiers, Poitiers, France
A. Kummert
Faculty of Electrical, Information and Media Engineering,
University of Wuppertal, Wuppertal, Germany 
pass $k$ acts as a forcing function on, and hence contributes to, the dynamics of the new pass profile $y_{k+1}(p), p=0,1, \ldots,(\alpha-1), k=0,1, \ldots$.

Industrial examples of repetitive processes include long-wall coal cutting operations and metal rolling operations (see the original papers cited in, e.g., Rogers \& Owens, 1992). Also a number of so-called algorithmic examples exist where adopting a repetitive process setting for analysis has clear advantages over alternative approaches to systems related analysis. These include iterative learning control schemes (Amann, Owens \& Rogers, 1998) and iterative solution algorithms for dynamic nonlinear optimal control problems based on the maximum principle (Roberts, 2002).

In the former case here, the sub-classes of so-called differential and discrete linear repetitive processes form the basis for a rigorous analysis of a powerful class of such algorithms. In the latter, the repetitive process setting for analysis has led to the development of numerically reliable and computationally efficient solution algorithms. Also, repetitive processes share many similarities with the so-called spatially interconnected systems, see e.g. (D'Andrea \& Dullerud, 2004), which are currently an active area of control system research with many potential applications areas, and hence repetitive process theory is also expected to find applications in this general area.

The essential unique control problem for these processes is that the output sequence of pass profiles generated can contain oscillations that increase in amplitude in the pass-to-pass (i.e., $k$ ) direction. Such behavior is easily generated in simulation studies and in experiments on scaled models of industrial processes such as long-wall coal cutting. In this last case, these oscillations are caused by the machine's weight as it comes to rest on the newly cut floor profile ready for the start of the next pass of the coal face.

A rigorous stability theory for linear repetitive processes has been developed. This theory (Rogers \& Owens, 1992) is based on an abstract model in a Banach space setting which includes a large number of such processes as special cases. This has results of applying this theory to a wide range of cases have been reported. This has resulted in stability tests for some sub-classes of practical interest that can, if desired, be implemented by direct application of well known $1 D$ linear systems tests. This theory consists of two distinct concepts termed asymptotic stability and stability along the pass respectively where the latter is a necessary condition for the former.

The fact that the pass length is finite (and hence information propagation in this direction only occurs over a finite duration) is a key difference with other classes of $2 D$ discrete linear systems. Also the initial conditions are reset before the start of each new pass and this can be quite complex, e.g., the initial conditions at the start of each new pass can be an explicit function of the previous pass profile. Also the structure of these alone can result in an unstable process (Rogers, Galkowski, Gramacki, Gramacki \& Owens, 2002) - a fact which has no counterpart in $1 D$ or $2 D$ systems theory. Overall, large parts of established systems theory for $2 D$ discrete linear systems described by the Roesser and Fornasini-Marchesini state-space models either cannot be applied at all or only after appropriate modifications have been made. Hence there is a need to develop a systems theory for these processes for onward translation (where appropriate) into numerically reliable algorithms.

Much of the work currently available on repetitive processes has focused on the definitions and characterizations of systems theoretic properties but recently the design of control schemes has become an active research area. For example, it is physically meaningful to define the current pass error as the difference, at each point along the pass, between a specified reference trajectory for that pass, which in most cases will be the same on each pass, and the actual pass profile produced. Then it is possible to define a so-called current pass error actuated controller which uses the generated error vector to construct the current pass 
control input vector. In which context, preliminary work, see, e.g., (Benton, 2000), has shown that, except in a few very restrictive special cases, the controller used must be actuated by a combination of information from the current and previous passes to guarantee even stability along the pass of the controlled process. The control action here is feedback of, for example, the current pass state or pass profile vector and feedforward (in the pass-to-pass direction) of previous pass information (most often the previous pass profile). (Note also that in the iterative learning control application area the previous pass (or trial) output is an obvious signal to use as feedforward action.) Design algorithms for such control laws applied to discrete linear repetitive processes can be found in, for example (Galkowski, Rogers, Xu, Lam, \& Owens, 2002).

Consider again the metal rolling operation. Then here a number of passes may be completed under one regime and then the dynamics change to allow further processing to take place. One way of modelling such a case is by switching the dynamics from one state-space model to an alternative (or alternatives). More generally, there are (at least) two distinct possibilities for switching dynamics to occur in repetitive processes; either the switching occurs from pass-to-pass or along a pass and this paper begins the development of tools for the analysis of these two cases. Both of these are practically motivated, e.g., switching from pass-to-pass can occur when handling multiple operation robot arms or multiple metal rolling systems, and along the pass switching can arise in the analysis of iterative learning control applied to processes with periodic dynamics. The new results developed are in the areas of applicable stability tests and the design of control laws activated by information measured on the current and previous passes.

The main idea underlying the derivation of the new results given in this paper is a form of lifting where similar approaches can be found in, for example, in iterative learning control analysis, e.g. (De Roover, Bosgra \& Steinbuch, 2000; Moore, 2000; Norrlof \& Gunnarsson, 2002; Rotariu, Dijkstra \& Steinbuch, 2004). This enables the analysis of parameter variant models to be replaced by that of parameter invariant models (but, obviously, of higher dimensions).

Throughout this paper, the null matrix and the identity matrix with the required dimensions are denoted by 0 and $I$, respectively. Moreover, $M>0(<0)$ denotes a real symmetric positive (negative) definite matrix.

\section{Background}

Discrete linear repetitive processes, which arise in both the iterative learning and optimal control application areas, are, in their basic form, defined by the state-space model

$$
\begin{aligned}
& x_{k+1}(p+1)=A x_{k+1}(p)+B u_{k+1}(p)+B_{0} y_{k}(p) \\
& y_{k+1}(p)=C x_{k+1}(p)+D u_{k+1}(p)+D 0 y_{k}(p) \\
& p=0,1, \ldots,(\alpha-1), \quad k=0,1, \ldots
\end{aligned}
$$

Here on pass $k, x_{k}(p)$ is the $n \times 1$ state vector, $y_{k}(p)$ is the $m \times 1$ pass profile vector, and $u_{k}(p)$ is the $r \times 1$ control input vector. To complete the process description, it is necessary to specify the boundary conditions, i.e., the state initial vector on each pass and the initial pass profile. Here no loss of generality arises from assuming $x_{k+1}(0)=d_{k+1}, k \geq 0$, and $y_{0}(p)=f(p)$, where the $n \times 1$ vector $d_{k+1}$ has known constant entries and $f(p)$ is an $m \times 1$ vector whose entries are known functions of $p$.

The abstract model based stability theory (Rogers \& Owens, 1992) for linear repetitive processes consists of two distinct concepts termed asymptotic stability and stability along 
the pass respectively. Noting again the unique control problem for these processes, this theory demands that bounded sequences of inputs produce bounded sequences of pass profiles where here bounded is defined in terms of the norm on the underlying function space. The essential difference between them is that asymptotic stability demands this property over the finite pass length whereas stability along the pass is stronger in that it demands this property uniformly, i.e., independent of the pass length.

In the case of processes described by (1), it can be shown (Rogers \& Owens, 1992) that asymptotic stability holds if, and only if, $r\left(D_{0}\right)<1$, where $r(\cdot)$ denotes the spectral radius of its matrix argument. Also if the example under consideration is asymptotically stable and the control input sequence applied $\left\{u_{k}\right\}_{k \geq 1}$ converges strongly to $u_{\infty}$ as $k \rightarrow \infty$ then the resulting output pass profile sequence $\left\{y_{k}\right\}_{k \geq 1}$ converges strongly to $y_{\infty}$ - the so-called limit profile-defined (with $D=0$ for ease of presentation) over $p=0,1, \ldots,(\alpha-1)$ by

$$
\begin{aligned}
& x_{\infty}(p+1)=\left(A+B_{0}\left(I-D_{0}\right)^{-1} C\right) x_{\infty}(p)+B u_{\infty}(p) \\
& y_{\infty}(p)=\left(I-D_{0}\right)^{-1} C x_{\infty}(p) .
\end{aligned}
$$

In effect, this result states that if a process is asymptotically stable then its repetitive dynamics can, after a 'sufficiently large' number of passes have elapsed, be replaced by those of a $1 D$ discrete linear system. Note, however, that this property does not guarantee that the limit profile is stable in the $1 D$ linear systems sense, i.e., $r\left(A+B_{0}\left(I-D_{0}\right)^{-1} C\right)<1-$ a point which is easily illustrated by, for example, the case when $A=-0.5, B=0, B_{0}=0.5+b_{0}$, $C=1, D=D_{0}=0$, and the real scalar $b_{0}$ is chosen such that $\left|b_{0}\right| \geq 1$.

Stability along the pass prevents cases such as the simple example above from arising (by demanding that the bound is independent of the pass length) and the following characterization is known (Rogers \& Owens, 1992).

Theorem 1 A discrete linear repetitive processes described by (1) is stable along the pass if, and only if, the so-called $2 D$ characteristic polynomial

$\mathcal{C}\left(z_{1}, z_{2}\right):=\operatorname{det}\left[\begin{array}{cc}I-z_{1} A & -z_{1} B_{0} \\ -z_{2} C & I-z_{2} D_{0}\end{array}\right]$

satisfies

$\mathcal{C}\left(z_{1}, z_{2}\right) \neq 0$ in $\bar{U}_{c}^{2}$

where $\bar{U}_{c}^{2}=\left\{\left(z_{1}, z_{2}\right):\left|z_{1}\right| \leq 1,\left|z_{2}\right| \leq 1\right\}$.

In theory, a repetitive process evolves over a semi-infinite strip in the positive quadrant of the $2 D$ domain, i.e., over $p=0,1, \ldots,(\alpha-1), k \geq 0$. Stability along the pass, however, treats the process as evolving over the complete positive quadrant, i.e. both $p$ and $k$ are of unbounded duration. For this reason, stability along the pass can be too strong in some cases of practical interest-see, for example, (Smyth, 1992) for further discussion of this point and illustrative examples. Also there are cases where asymptotic stability is all that can be achieved, e.g., the optimal control application (Roberts, 2002).

A similar situation arises in class of $2 D$ discrete linear systems which includes those described by the Roesser and Fornasini-Marchesini state-space models as special cases, and this has led to the concept of so-called practical stability first introduced in (Agathoklis \& Bruton, 1983). To explain the motivation for this, it is instructive to briefly consider the $1 D$ case, i.e., a discrete linear system with input and output sequences denoted by $u(i)$ and $y(i)$ respectively. Then the standard definition of bounded-input bounded-output (BIBO) stability demands that the output sequence $y(i)$ is bounded in amplitude for all input signals that are 
bounded in amplitude (here the term bounded in amplitude is interpreted in terms of the norm on the underlying function space). Note, however, that the input and output signals may be of unbounded duration, i.e., $i$ may be unbounded, and in many cases $i$ is a temporal variable.

The $1 D$ definition of BIBO stability has been extended to the $2 D$ (and also the $n D, n \geq 3$ ) case by considering input signals which are unbounded in both variables. The basic idea of practical stability in the sense of (Agathoklis \& Bruton, 1983) for $2 D$ systems is to consider BIBO stability when the input signals are of unbounded duration in at most one variable. To characterize practical stability for discrete linear repetitive processes, first note again that $r\left(D_{0}\right)<1$, i.e., asymptotic stability is a necessary condition for stability along the pass. Also consideration of the current pass dynamics alone, i.e., with the previous pass terms in the first equation of (1) deleted, leads immediately to $r(A)<1$ as another necessary condition for stability along the pass. The conditions $r\left(D_{0}\right)<1$ and $r(A)<1$ can be regarded as a weak form of practical stability-here simply termed practical stability and defined formally as follows.

Definition 1 A discrete linear repetitive process described by (1) is said to be practically stable when $r\left(D_{0}\right)<1$ and $r(A)<1$.

Note As the simple example given earlier, i.e., $A=-0.5, B=0, B_{0}=0.5+b_{0}, C=$ $1, D=D_{0}=0,\left|b_{0}\right| \geq 1$, demonstrates, practical stability is also only a necessary condition for stability along the pass.

Previous work, see, for example (Galkowski et al., 2002), has used an LMI setting to design control laws of the following form over $p=0,1, \ldots,(\alpha-1), k=0,1, \ldots$ for processes described by (1)

$u_{k+1}(p)=K_{1} x_{k+1}(p)+K_{2} y_{k}(p)=: K\left[\begin{array}{c}x_{k+1}(p) \\ y_{k}(p)\end{array}\right]$

where $K_{1}$ and $K_{2}$ are appropriately dimensioned matrices to be designed. In effect, this control law uses feedback of the current state vector (which is assumed to be available for use) and feedforward of the previous pass profile vector.

\section{Discrete linear repetitive processes with dynamics switched from pass-to-pass}

All of the work which has been reported on the analysis and control of discrete repetitive processes either assumes that once the state-space model is obtained it remains fixed for the complete duration of passes or (more recently) that it is subject to well defined uncertainty structures. In some cases, however, a more realistic scenario is that there a number of regimes of operation, each of which has a state-space model description and, in operation, the process switches between them according to some given schedule. For example, in metal rolling, it may be required to pass the workpiece through a series of passes which are described by different state-space models, i.e., complete a number of passes with one model in place and then switch to complete another number of passes described by a different model and so on.

The dynamics in the scenario described above switch in the pass-to-pass direction and we now undertake the first analysis of this case where it is assumed that the process dynamics are described as follows for $p=0,1, \ldots,(\alpha-1), l=0,1, \ldots$

$$
\begin{gathered}
x_{l+1}(p+1)=\left\{\begin{array}{l}
A_{1} x_{l+1}(p)+B_{1} u_{l+1}(p)+B_{01} y_{l}(p), \text { for } l=0,2, \ldots, \text { i.e., } l=2 k, \\
A_{2} x_{l+1}(p)+B_{2} u_{l+1}(p)+B_{02} y_{l}(p), \text { for } l=1,3, \ldots, \text { i.e., } l=2 k+1
\end{array}\right. \\
y_{l+1}(p)=\left\{\begin{array}{l}
C_{1} x_{l+1}(p)+D_{1} u_{l+1}(p)+D_{01} y_{l}(p), \text { for } l=0,2, \ldots, \text { i.e., } l=2 k \\
C_{2} x_{l+1}(p)+D_{2} u_{l+1}(p)+D_{02} y_{l}(p), \text { for } l=1,3, \ldots, \text { i.e., } l=2 k+1
\end{array}\right.
\end{gathered}
$$


where the state, pass profile, and control input vectors together with the boundary conditions are as for processes described by (1). This model assumes that the dynamics switch on completion of each pass profile and is clearly not the most general case but, given the absence of any previous results in this area, it will act as a starting point with the possibility that the experience gained will lead to straightforward generalizations to other cases.

One obvious approach to the analysis of the process model given above is to attempt to transform it into an equivalent model of the form (1) and then directly apply the existing results. Introduce, therefore, the following new state, pass profile and input vectors

$X_{l+1}(p)=\left[\begin{array}{l}x_{2 k+1}(p) \\ x_{2 k+2}(p)\end{array}\right], \quad U_{l+1}(p)=\left[\begin{array}{l}u_{2 k+1}(p) \\ u_{2 k+2}(p)\end{array}\right], \quad Y_{l}(p)=y_{2 k}(p)$,

$Y_{l+1}(p)=y_{2 k+2}(p)$

Then the equivalent model of the form (1) for processes described by (2) is

$X_{l+1}(p+1)=\widehat{A} X_{l+1}(p)+\widehat{B} U_{l+1}(p)+\widehat{B}_{0} Y_{l}(p)$

$Y_{l+1}(p)=\widehat{C} X_{l+1}(p)+\widehat{D} U_{l+1}(p)+\widehat{D}_{0} Y_{l}(p)$

where

$$
\begin{aligned}
& \widehat{A}=\left[\begin{array}{cc}
A_{1} & 0 \\
B_{02} C_{1} & A_{2}
\end{array}\right], \quad \widehat{B}=\left[\begin{array}{cc}
B_{1} & 0 \\
B_{02} D_{1} & B_{2}
\end{array}\right], \quad \widehat{B}_{0}=\left[\begin{array}{c}
B_{01} \\
B_{02} D_{01}
\end{array}\right] \\
& \widehat{C}=\left[\begin{array}{lll}
D_{02} C_{1} C_{2}
\end{array}\right], \quad \widehat{D}=\left[\begin{array}{ll}
D_{02} D_{1} D_{2}
\end{array}\right], \quad \widehat{D}_{0}=D_{02} D_{01}
\end{aligned}
$$

This model has some links with the lifting approach to ILC analysis referred to in the introduction section of this paper but here lifting is applied in the pass-to-pass direction with the effect of combining successive even and odd pass numbers.

Now it is possible to give conditions for stability of processes described by (2). We start with asymptotic stability or practical stability where the following result is immediate from application of the conditions for these properties to hold for processes described by (1).

Theorem 2 A discrete linear repetitive process described by (2) is:

(i) asymptotically stable if, and only if, $r\left(D_{02} D_{01}\right)<1$; and

(ii) practically stable if, and only if, $r\left(D_{02} D_{01}\right)<1, r\left(A_{1}\right)<1$, and $r\left(A_{2}\right)<1$.

Proof Follows immediately on applying the respective stability conditions to the lifted model (3).

Also it is possible to obtain the conditions for stability along the pass. Of the numerous sets of conditions which have been developed, the most relevant here is the following one (Galkowski et al., 2002) based on the use of LMIs. This condition is sufficient, and hence the possibility of a significant degree of conservativeness, but, unlike necessary and sufficient alternatives, leads easily to control law design algorithms (as shown below). As a preliminary step, it is convenient to introduce the following matrices from (3) (and hence (2)).

$\widehat{A}_{1}=\left[\begin{array}{cc}\widehat{A} & \widehat{B}_{0} \\ 0 & 0\end{array}\right], \quad \widehat{A}_{2}=\left[\begin{array}{cc}0 & 0 \\ \widehat{C} & \widehat{D}_{0}\end{array}\right]$

Then we have the following sufficient condition for stability along the pass of processes described by (2).

Theorem 3 (Galkowski et al., 2002) A discrete linear repetitive process described by (2) is stable along the pass if $\exists$ matrices $P>0$ and $Q>0$ satisfying the following $L M I$

$\left[\begin{array}{cc}\widehat{A}_{1}^{T} P \widehat{A}_{1}+Q-P & \widehat{A}_{1}^{T} P \widehat{A}_{2} \\ \widehat{A}_{2}^{T} P \widehat{A}_{1} & \widehat{A}_{2}^{T} P \widehat{A}_{2}-Q\end{array}\right]<0$

Springer 
The following corollary (Galkowski et al., 2002) to this last result will also be used in the analysis which follows.

Corollary 1 A discrete linear repetitive process described by (2) is stable along the pass if $\exists$ matrices $W_{1}>0$ and $W_{2}>0$ such that

$\widehat{\Phi}^{T} W \widehat{\Phi}-W<0$

holds, where $\widehat{\Phi}$ is the so-called augmented plant matrix defined by

$\widehat{\Phi}=\left[\begin{array}{ll}\widehat{A} & \widehat{B}_{0} \\ \widehat{C} & \widehat{D}_{0}\end{array}\right]=\widehat{A}_{1}+\widehat{A}_{2}$

and $W=W_{1} \oplus W_{2}$ with $\oplus$ denoting the direct sum, i.e., $W=\operatorname{diag}\left\{W_{1}, W_{2}\right\}$.

Note also that (5) is (one formulation of) the so-called $2 D$ Lyapunov equation for a process described by (1) (Galkowski et al., 2002).

As a first attempt to control these processes, we consider a switched control law of the form

$u_{l+1}(p)=\left\{\begin{array}{l}K_{1}^{1} x_{l+1}(p)+K_{2}^{1} y_{l}(p), \text { for } l=0,2, \ldots, \text { i.e., } l=2 k \\ K_{1}^{2} x_{l+1}(p)+K_{2}^{2} y_{l}(p), \text { for } l=1,3, \ldots, \text { i.e., } l=2 k+1\end{array}\right.$

or, in lifted form,

$U_{l+1}(p)=\widehat{K}_{1} X_{l+1}(p)+\widehat{K}_{2} Y_{l}(p)$

where

$\widehat{K}_{1}=\left[\begin{array}{cc}K_{1}^{1} & 0 \\ K_{2}^{2}\left(C_{1}+D_{1} K_{1}^{1}\right) & K_{1}^{2}\end{array}\right], \quad \widehat{K}_{2}=\left[\begin{array}{c}K_{2}^{1} \\ K_{2}^{2}\left(D_{01}+D_{1} K_{2}^{1}\right)\end{array}\right]$

The controlled process state-space model can be written in lifted form as

$$
\begin{gathered}
X_{l+1}(p+1)=\widehat{A}_{\text {new }} X_{l+1}(p)+\widehat{B}_{\text {0new }} Y_{l}(p) \\
Y_{l+1}(p)=\widehat{C}_{\text {new }} X_{l+1}(p)+\widehat{D}_{\text {0new }} Y_{l}(p)
\end{gathered}
$$

where

$$
\begin{aligned}
& \widehat{A}_{\text {new }}=\widehat{A}+\widehat{B} \widehat{K}_{1}=\left[\begin{array}{cc}
A_{1}+B_{1} K_{1}^{1} & 0 \\
\left(B_{02}+B_{2} K_{2}^{2}\right)\left(C_{1}+D_{1} K_{1}^{1}\right) & A_{2}+B_{2} K_{1}^{2}
\end{array}\right] \\
& \widehat{B}_{0 \text { new }}=\widehat{B}_{0}+\widehat{B} \widehat{K}_{2}=\left[\begin{array}{c}
B_{01}+B_{1} K_{2}^{1} \\
\left(B_{02}+B_{2} K_{2}^{2}\right)\left(D_{01}+D_{1} K_{2}^{1}\right)
\end{array}\right] \\
& \widehat{C}_{n e w}=\widehat{C}+\widehat{D} \widehat{K}_{1}=\left[\left(D_{02}+D_{2} K_{2}^{2}\right)\left(C_{1}+D_{1} K_{1}^{1}\right) C_{2}+D_{2} K_{1}^{2}\right] \\
& \widehat{D}_{\text {0new }}=\widehat{D}_{0}+\widehat{D} \widehat{K}_{2}=\left(D_{02}+D_{2} K_{2}^{2}\right)\left(D_{01}+D_{1} K_{2}^{1}\right)
\end{aligned}
$$

Also introduce

$\widehat{\Phi}_{\text {new }}=\left[\begin{array}{ll}\widehat{A}_{\text {new }} & \widehat{B}_{\text {0new }} \\ \widehat{C}_{\text {new }} & \widehat{D}_{\text {Onew }}\end{array}\right]=\left[\begin{array}{ll}\widehat{A} & \widehat{B}_{0} \\ \widehat{C} & \widehat{D}_{0}\end{array}\right]+\left[\begin{array}{l}\widehat{B} \\ \widehat{D}\end{array}\right]\left[\begin{array}{ll}\widehat{K}_{1} & \widehat{K}_{2}\end{array}\right]$

and

$$
\widehat{\Phi}_{\text {new }}=\left[\begin{array}{ccc}
A_{1}+B_{1} K_{1}^{1} & 0 & B_{01}+B_{1} K_{2}^{1} \\
\left(B_{02}+B_{2} K_{2}^{2}\right)\left(C_{1}+D_{1} K_{1}^{1}\right) & A_{2}+B_{2} K_{1}^{2} & \left(B_{02}+B_{2} K_{2}^{2}\right)\left(D_{01}+D_{1} K_{2}^{1}\right) \\
\left(D_{02}+D_{2} K_{2}^{2}\right)\left(C_{1}+D_{1} K_{1}^{1}\right) & C_{2}+D_{2} K_{1}^{2} & \left(D_{02}+D_{2} K_{2}^{2}\right)\left(D_{01}+D_{1} K_{2}^{1}\right)
\end{array}\right]
$$


Then we can write $\widehat{\Phi}_{n e w}$ in the more compact form

$\widehat{\Phi}_{n e w}=\Phi_{1}+\Phi_{2}=\Phi_{1}+\Phi_{2}^{1} \Phi_{2}^{2}$

where

$\Phi_{1}=\bar{A}_{1}+\bar{B}_{1} \bar{K}_{1}, \quad \Phi_{2}^{1}=\bar{A}_{2}^{1}+\bar{B}_{2}^{1} \bar{K}_{2}, \quad \Phi_{2}^{2}=\bar{A}_{2}^{2}+\bar{B}_{2}^{2} \bar{K}_{1}$

and

$$
\begin{aligned}
& \bar{A}_{1}=\left[\begin{array}{ccc}
A_{1} & 0 & B_{01} \\
0 & A_{2} & 0 \\
0 & C_{2} & 0
\end{array}\right], \quad \bar{B}_{1}=\left[\begin{array}{ccc}
B_{1} & 0 & B_{1} \\
0 & B_{2} & 0 \\
0 & D_{2} & 0
\end{array}\right], \quad \bar{K}_{1}=\left[\begin{array}{ccc}
K_{1}^{1} & 0 & 0 \\
0 & K_{1}^{2} & 0 \\
0 & 0 & K_{2}^{1}
\end{array}\right] \\
& \bar{A}_{2}^{1}=\left[\begin{array}{ccc}
0 & 0 & 0 \\
0 & B_{02} & 0 \\
0 & 0 & D_{02}
\end{array}\right], \quad \bar{B}_{2}^{1}=\left[\begin{array}{ccc}
0 & 0 & 0 \\
0 & B_{2} & 0 \\
0 & 0 & D_{2}
\end{array}\right], \quad \bar{K}_{2}=\left[\begin{array}{ccc}
K_{2}^{2} & 0 & 0 \\
0 & K_{2}^{2} & 0 \\
0 & 0 & K_{2}^{2}
\end{array}\right] \\
& \bar{A}_{2}^{2}=\left[\begin{array}{ccc}
0 & 0 & 0 \\
C_{1} & 0 & D_{01} \\
C_{1} & 0 & D_{01}
\end{array}\right], \quad \bar{B}_{2}^{2}=\left[\begin{array}{ccc}
0 & 0 & 0 \\
D_{1} & 0 & D_{1} \\
D_{1} & 0 & D_{1}
\end{array}\right]
\end{aligned}
$$

\subsection{Stabilization to achieve asymptotic and practical stability}

As noted previously, there are cases where asymptotic stability is all that can be achieved and others where stability along the pass is most appropriately replaced by the weaker condition of practical stability. In the analysis which follows, we develop computable conditions under which these properties can be achieved using a control law of the form (6).

Theorem 4 Suppose that a control law with the structure of (6) is applied a discrete linear repetitive process which can be written in the form (3). Then the resulting process is asymptotically stable if, and only if, $\exists$ a matrix $X>0$, two nonsingular matrices $V$ and $G$ and two matrices $N_{1}$ and $N_{2}$ such that the following LMI holds

$$
\left[\begin{array}{cccc}
-X & D_{02} V+D_{2} N_{2} & 0 & 0 \\
\left(D_{02} V+D_{2} N_{2}\right)^{T} & -V-V^{T} & 0 & D_{01} G+D_{1} N_{1} \\
0 & 0 & -X & X \\
0 & \left(D_{01} G+D_{1} N_{1}\right)^{T} & X^{T} & -G-G^{T}
\end{array}\right]<0
$$

If this condition holds, the matrices in the control law (6) are given by

$K_{2}^{1}=N_{1} G^{-1}, \quad K_{2}^{2}=N_{2} V^{-1}$

and $K_{1}^{1}=K_{1}^{2}=0$. Also practically stability holds if and only if (7) and (8) hold and $\exists$ matrices $X_{i}>0$, nonsingular $V_{i}$, and matrices $M_{i}, i=1,2$ such that the following $L M I$ holds

$\left[\begin{array}{cc}-X_{i} & A_{i} V_{i}+B_{i} M_{i} \\ \left(A_{i} V_{i}+B_{i} M_{i}\right)^{T} & X_{i}-V_{i}-V_{i}^{T}\end{array}\right]<0, \quad i=1,2$

If this condition holds, the corresponding control law matrices are given by

$K_{1}^{i}=M_{i} V_{i}^{-1}, \quad i=1,2$

and $K_{2}^{i}, i=1,2$ are as in the asymptotic stability case.

烂 Springer 
Proof Follows immediately on using Theorem 3 and known LMI results for $1 D$ linear systems considered in (Mehdi, Bachelier \& Galkowski, 2004) and (Peaucelle, Arzelier, Bachelier \& Bernussou, 2000).

Remark The reason why the above result gives necessary and sufficient conditions is that the properties concerned are completely expressed in terms of conditions on the spectral radius of the matrices $\widehat{A}$ and $\widehat{D}_{0}$ respectively from the $1 D$ equivalent model and hence in both cases were are dealing with the $1 D$ discrete linear systems stability condition. When we consider control law design for stability along the pass (next sub-section) this is will not be the case.

\subsection{Stabilization to achieve stability along the pass}

We will require the following lemma to establish the main result of this section in the form of Theorem 5 below.

Lemma 1 If there exist such appropriately dimensioned matrices $U<0, O<0$ and $F$ such that

$\left[\begin{array}{cc}U & F \\ F^{T} & O\end{array}\right]<0$

then

$\Gamma:=U+O+F+F^{T}<0$

Proof Immediate on noting that

$\left[\begin{array}{ll}I & I\end{array}\right]\left[\begin{array}{cc}U & F \\ F^{T} & O\end{array}\right]\left[\begin{array}{l}I \\ I\end{array}\right]=\Gamma$

Remark The matrix [ I I ] is rectangular and hence not of maximal rank, i.e., not a congruence, and hence it is not possible to establish the converse implication in this last result. This, in turn, could result in an increased degree of conservativeness in subsequent analysis. Now we have the following result.

Theorem 5 Suppose that a control law with the structure of (6) is applied to a discrete linear repetitive process which can be written in the form (3). Then the resulting process is stable along the pass, if $\exists$ matrices $X_{i}>0, i=1,2, Y>0$, square matrices $V_{i}, i=1,2,3$ and $Z$, and rectangular matrices $L_{i}, i=1,2,3$ and $N$ such that

$\left[\begin{array}{cccccc}-X+Y & 0 & \bar{A}_{1} \bar{V}+\bar{B}_{1} \bar{L} & 0 & 0 & 0 \\ 0 & -X-Y & 0 & \bar{A}_{2}^{1} \bar{Z}+\bar{B}_{2}^{1} \bar{N} & 0 & 0 \\ \bar{V}^{T} \bar{A}_{1}^{T}+\bar{L}^{T} \bar{B}_{1}^{T} & 0 & -\bar{V}-\bar{V}^{T} & 0 & X & 0 \\ 0 & \bar{Z}^{T} \bar{A}_{2}^{1 T}+\bar{N}^{T} \bar{B}_{2}^{1 T} & 0 & -\bar{Z}-\bar{Z}^{T} & \bar{A}_{2}^{2} \bar{V}+\bar{B}_{2}^{2} \bar{L} & \bar{A}_{2}^{2} \bar{V}+\bar{B}_{2}^{2} \bar{L} \\ 0 & 0 & X & \bar{V}^{T} \bar{A}_{2}^{2 T}+\bar{L}^{T} \bar{B}_{2}^{2 T} & -X & 0 \\ 0 & 0 & 0 & \bar{V}^{T} \bar{A}_{2}^{2 T}+\bar{L}^{T} \bar{B}_{2}^{2 T} & 0 & X-\bar{V}-\bar{V}^{T}\end{array}\right]<0$

where

$$
\begin{aligned}
& X=\left[\begin{array}{cc}
X_{1} & 0 \\
0 & X_{2}
\end{array}\right], \quad \bar{V}=\left[\begin{array}{ccc}
V_{1} & 0 & 0 \\
0 & V_{2} & 0 \\
0 & 0 & V_{3}
\end{array}\right], \quad \bar{Z}=\left[\begin{array}{lll}
Z & 0 & 0 \\
0 & Z & 0 \\
0 & 0 & Z
\end{array}\right] \\
& \bar{L}=\left[\begin{array}{ccc}
L_{1} & 0 & 0 \\
0 & L_{2} & 0 \\
0 & 0 & L_{3}
\end{array}\right], \quad \bar{N}=\left[\begin{array}{ccc}
N & 0 & 0 \\
0 & N & 0 \\
0 & 0 & N
\end{array}\right]
\end{aligned}
$$


If this condition holds, the control law matrices are given by

$$
\bar{K}_{1}=\bar{L} \bar{V}^{-1}=\left[\begin{array}{ccc}
K_{1}^{1} & 0 & 0 \\
0 & K_{1}^{2} & 0 \\
0 & 0 & K_{2}^{1}
\end{array}\right], \quad \bar{K}_{2}=\bar{N} \bar{Z}^{-1}=\left[\begin{array}{ccc}
K_{2}^{2} & 0 & 0 \\
0 & K_{2}^{2} & 0 \\
0 & 0 & K_{2}^{2}
\end{array}\right]
$$

Proof Applying Corollary 1 to this gives that stability along the pass holds if $\exists$ a matrix $X=\operatorname{diag}\left\{X_{1}, X_{2}\right\}>0$ such that

$\widehat{\Phi}_{\text {new }} X \widehat{\Phi}_{\text {new }}^{T}-X<0$

or, equivalently,

$\Phi_{1} X \Phi_{1}^{T}+\Phi_{1} X \Phi_{2}^{T}+\Phi_{2} X \Phi_{1}^{T}+\Phi_{2} X \Phi_{2}^{T}-X<0$

Now write

$\left(\Phi_{1} X \Phi_{1}^{T}-X+Y\right)+\left(\Phi_{2} X \Phi_{1}^{T}\right)+\left(\Phi_{1} X \Phi_{2}^{T}\right)+\left(\Phi_{2} X \Phi_{2}^{T}-Y\right)<0$

where $Y>0$ has the same dimensions as $X$. Then by Lemma 1 this last inequality holds provided

$$
\left[\begin{array}{cc}
\Phi_{1} X \Phi_{1}^{T}-X+Y & \Phi_{1} X \Phi_{2}^{T} \\
\Phi_{2} X \Phi_{1}^{T} & \Phi_{2} X \Phi_{2}^{T}-Y
\end{array}\right]<0
$$

or, equivalently,

$$
\left[\begin{array}{l}
\Phi_{1} \\
\Phi_{2}
\end{array}\right] X\left[\begin{array}{ll}
\Phi_{1}^{T} & \Phi_{2}^{T}
\end{array}\right]+\left[\begin{array}{cc}
-X+Y & 0 \\
0 & -Y
\end{array}\right]<0
$$

An obvious application of the Schur's complement formula to this last expression now yields

$$
\left[\begin{array}{ccc}
-X+Y & 0 & \Phi_{1} \\
0 & -Y & \Phi_{2} \\
\Phi_{1}^{T} & \Phi_{2}^{T} & -X^{-1}
\end{array}\right]<0
$$

Next, pre-and post-multiply this last expression by $\left[\begin{array}{ccc}I & 0 & 0 \\ 0 & I & 0 \\ 0 & 0 & \bar{V}\end{array}\right]^{T}$ and its transpose respectively to yield

$$
\left[\begin{array}{ccc}
-X+Y & 0 & \Phi_{1} \bar{V} \\
0 & -Y & \Phi_{2} \bar{V} \\
\bar{V}^{T} \Phi_{1}^{T} & \bar{V}^{T} \Phi_{2}^{T} & -\bar{V}^{T} X^{-1} \bar{V}
\end{array}\right]<0
$$

Also, since $(X-\bar{V})^{T} X^{-1}(X-\bar{V})>0,-\bar{V}^{T} X^{-1} \bar{V}<X-\bar{V}-\bar{V}^{T}$ and hence we can write

$$
\left[\begin{array}{ccc}
-X+Y & 0 & \Phi_{1} \bar{V} \\
0 & -Y & \Phi_{2} \bar{V} \\
\bar{V}^{T} \Phi_{1}^{T} & \bar{V}^{T} \Phi_{2}^{T} & X-\bar{V}-\bar{V}^{T}
\end{array}\right]<0
$$

i.e.,

$$
\left.\begin{array}{ccc}
-X+Y & 0 & \Phi_{1} \bar{V} \\
0 & -Y & \Phi_{2}^{1} \Phi_{2}^{2} \bar{V} \\
\bar{V}^{T} \Phi_{1}^{T} & \bar{V}^{T} \Phi_{2}^{2 T} \Phi_{2}^{1 T} & X-\bar{V}-\bar{V}^{T}
\end{array}\right]<0
$$


Substituting for $\Phi_{2}$ now yields

$$
\begin{aligned}
& {\left[\begin{array}{ccc}
-X+Y & 0 & \Phi_{1} \bar{V} \\
0 & -Y-\Phi_{2}^{1} \Phi_{2}^{2} X \Phi_{2}^{2 T} \Phi_{2}^{1 T} & 0 \\
\bar{V}^{T} \Phi_{1}^{T} & 0 & -\bar{V}-\bar{V}^{T}
\end{array}\right]} \\
& +\left[\begin{array}{ccc}
0 & 0 & 0 \\
0 & \Phi_{2}^{1} & 0 \\
0 & 0 & I
\end{array}\right]\left[\begin{array}{ccc}
X & 0 & 0 \\
0 & \Phi_{2}^{2} X \Phi_{2}^{2 T} & \Phi_{2}^{2} \bar{V} \\
0 & \bar{V}^{T} \Phi_{2}^{2 T} & X
\end{array}\right]\left[\begin{array}{ccc}
0 & 0 & 0 \\
0 & \Phi_{2}^{1} & 0 \\
0 & 0 & I
\end{array}\right]^{T}<0
\end{aligned}
$$

Now introduce

$$
\begin{aligned}
& \mathbf{W}=\left[\begin{array}{ccc}
-X+Y & 0 & \Phi_{1} \bar{V} \\
0 & -Y-\Phi_{2}^{1} \Phi_{2}^{2} X \Phi_{2}^{2 T} \Phi_{2}^{1 T} & 0 \\
\bar{V}^{T} \Phi_{1}^{T} & 0 & -\bar{V}-\bar{V}^{T}
\end{array}\right] \\
& \mathbf{L}=\left[\begin{array}{lll}
0 & 0 & 0 \\
0 & \Phi_{2}^{1} & 0 \\
0 & 0 & I
\end{array}\right], \quad \mathbf{V}=\left[\begin{array}{ccc}
X & 0 & 0 \\
0 & \Phi_{2}^{2} X \Phi_{2}^{2 T} & \Phi_{2}^{2} \bar{V} \\
0 & \bar{V}^{T} \Phi_{2}^{2 T} & X
\end{array}\right] \\
& \overline{\mathbf{V}}=\left[\begin{array}{lll}
X & 0 & 0 \\
0 & \bar{Z} & 0 \\
0 & 0 & X
\end{array}\right]
\end{aligned}
$$

where $\bar{Z}=I_{3} \otimes Z$, and $\otimes$ denotes the matrix Kronecker product. Then we can write the previous inequality in the compact form $\mathbf{W}+\mathbf{L}^{T} \mathbf{V L}<0$ with $\mathbf{V}>0$ and $\mathbf{W}=\mathbf{W}^{T}$ which, on completing an obvious application of the Schur's complement formula, is equivalent to

$\left[\begin{array}{cc}\mathbf{W} & \mathbf{L}^{T} \\ \mathbf{L} & -\mathbf{V}^{-1}\end{array}\right]<0$

and subsequent use of an appropriate congruence transformation yields

$$
\left[\begin{array}{cc}
\mathbf{W} & \mathbf{L}^{T} \overline{\mathbf{V}} \\
\overline{\mathbf{V}}^{T} \mathbf{L}-\overline{\mathbf{V}}^{T} \mathbf{V}^{-1} \overline{\mathbf{V}}
\end{array}\right]<0
$$

Next, since $-\overline{\mathbf{V}}^{T} \mathbf{V}^{-1} \overline{\mathbf{V}}<\mathbf{V}-\overline{\mathbf{V}}-\overline{\mathbf{V}}^{T}$, we have that

$$
\left[\begin{array}{cc}
\mathbf{W} & \mathbf{L}^{T} \overline{\mathbf{V}} \\
\overline{\mathbf{V}}^{T} \mathbf{L} \mathbf{V}-\overline{\mathbf{V}}-\overline{\mathbf{V}}^{T}
\end{array}\right]<0
$$

and this last inequality can be expressed as

$$
\left[\begin{array}{cccccc}
-X+Y & 0 & \Phi_{1} \bar{V} & 0 & 0 & 0 \\
0 & -Y-\Phi_{2}^{1} \Phi_{2}^{2} X \Phi_{2}^{2 T} \Phi_{2}^{1 T} & 0 & 0 & \Phi_{2}^{1} \bar{Z} & 0 \\
\bar{V}^{T} \Phi_{1}^{T} & 0 & -\bar{V}-\bar{V}^{T} & 0 & 0 & X \\
0 & 0 & 0 & -X & 0 & 0 \\
0 & \bar{Z}^{T} \Phi_{2}^{1 T} & 0 & 0 & \Phi_{2}^{2} X \Phi_{2}^{2 T}-\bar{Z}-\bar{Z}^{T} & \Phi_{2}^{2} \bar{V} \\
0 & 0 & X & 0 & \bar{V}^{T} \Phi_{2}^{2 T} & -X
\end{array}\right]<0
$$

Also, since $-Y-\Phi_{2}^{1} \Phi_{2}^{2} X \Phi_{2}^{2 T} \Phi_{2}^{1 T}=-Y-\Phi_{2} X \Phi_{2}^{T}<-Y-X$, this last matrix inequality can be rewritten as

$$
\left[\begin{array}{cccccc}
-X+Y & 0 & \Phi_{1} \bar{V} & 0 & 0 & 0 \\
0 & -X-Y & 0 & 0 & \Phi_{2}^{1} \bar{Z} & 0 \\
\bar{V}^{T} \Phi_{1}^{T} & 0 & -\bar{V}-\bar{V}^{T} & 0 & 0 & X \\
0 & 0 & 0 & -X & 0 & 0 \\
0 & \bar{Z}^{T} \Phi_{2}^{1 T} & 0 & 0 & \Phi_{2}^{2} X \Phi_{2}^{2 T}-\bar{Z}-\bar{Z}^{T} & \Phi_{2}^{2} \bar{V} \\
0 & 0 & X & 0 & \bar{V}^{T} \Phi_{2}^{2 T} & -X
\end{array}\right]<0
$$

or, equivalently, 


$$
\begin{aligned}
& {\left[\begin{array}{cccccc}
-X+Y & 0 & \Phi_{1} \bar{V} & 0 & 0 & 0 \\
0 & -X-Y & 0 & 0 & \Phi_{2}^{1} \bar{Z} & 0 \\
\bar{V}^{T} \Phi_{1}^{T} & 0 & -\bar{V}-\bar{V}^{T} & 0 & 0 & X \\
0 & 0 & 0 & -X & 0 & 0 \\
0 & \bar{Z}^{T} \Phi_{2}^{1 T} & 0 & 0 & -\bar{Z}-\bar{Z}^{T} & \Phi_{2}^{2} \bar{V} \\
0 & 0 & X & 0 & \bar{V}^{T} \Phi_{2}^{2 T} & -X
\end{array}\right]} \\
& +\left[\begin{array}{llllll}
0 & 0 & 0 & 0 & 0 & 0 \\
0 & 0 & 0 & 0 & 0 & 0 \\
0 & 0 & 0 & 0 & 0 & 0 \\
0 & 0 & 0 & 0 & 0 & 0 \\
0 & 0 & 0 & 0 & \Phi_{2}^{2} & 0 \\
0 & 0 & 0 & 0 & 0 & 0
\end{array}\right]\left[\begin{array}{cccccc}
X & 0 & 0 & 0 & 0 & 0 \\
0 & X & 0 & 0 & 0 & 0 \\
0 & 0 & X & 0 & 0 & 0 \\
0 & 0 & 0 & X & 0 & 0 \\
0 & 0 & 0 & 0 & X & 0 \\
0 & 0 & 0 & 0 & 0 & X
\end{array}\right]\left[\begin{array}{llllll}
0 & 0 & 0 & 0 & 0 & 0 \\
0 & 0 & 0 & 0 & 0 & 0 \\
0 & 0 & 0 & 0 & 0 & 0 \\
0 & 0 & 0 & 0 & 0 & 0 \\
0 & 0 & 0 & 0 & \Phi_{2}^{2} & 0 \\
0 & 0 & 0 & 0 & 0 & 0
\end{array}\right]^{T}<0
\end{aligned}
$$

By the Schur's complement formula, and deleting superfluous rows and columns, we now have that

$$
\left[\begin{array}{cccccc}
-X+Y & 0 & \Phi_{1} \bar{V} & 0 & 0 & 0 \\
0 & -X-Y & 0 & \Phi_{2}^{1} \bar{Z} & 0 & 0 \\
\bar{V}^{T} \Phi_{1}^{T} & 0 & -\bar{V}-\bar{V}^{T} & 0 & X & 0 \\
0 & \bar{Z}^{T} \Phi_{2}^{1 T} & 0 & -\bar{Z}-\bar{Z}^{T} & \Phi_{2}^{2} \bar{V} & \Phi_{2}^{2} \\
0 & 0 & X & \bar{V}^{T} \Phi_{2}^{2 T} & -X & 0 \\
0 & 0 & 0 & \Phi_{2}^{2 T} & 0 & -X^{-1}
\end{array}\right]<0
$$

Pre- and post-multiplying this last expression by

$$
\left[\begin{array}{llllll}
I & 0 & 0 & 0 & 0 & 0 \\
0 & I & 0 & 0 & 0 & 0 \\
0 & 0 & I & 0 & 0 & 0 \\
0 & 0 & 0 & I & 0 & 0 \\
0 & 0 & 0 & 0 & I & 0 \\
0 & 0 & 0 & 0 & 0 & \bar{V}
\end{array}\right]^{T}
$$

and its transpose respectively now yields

$$
\left[\begin{array}{cccccc}
-X+Y & 0 & \Phi_{1} \bar{V} & 0 & 0 & 0 \\
0 & -X-Y & 0 & \Phi_{2}^{1} \bar{Z} & 0 & 0 \\
\bar{V}^{T} \Phi_{1}^{T} & 0 & -\bar{V}-\bar{V}^{T} & 0 & X & 0 \\
0 & \bar{Z}^{T} \Phi_{2}^{1 T} & 0 & -\bar{Z}-\bar{Z}^{T} & \Phi_{2}^{2} \bar{V} & \Phi_{2}^{2} \bar{V} \\
0 & 0 & X & \bar{V}^{T} \Phi_{2}^{2 T} & -X & 0 \\
0 & 0 & 0 & \bar{V}^{T} \Phi_{2}^{2 T} & 0 & -\bar{V}^{T} X^{-1} \bar{V}
\end{array}\right]<0
$$

or, since $(X-\bar{V})^{T} X^{-1}(X-\bar{V})>0$ and hence $-\bar{V}^{T} X^{-1} \bar{V}<X-\bar{V}-\bar{V}^{T}$,

$$
\left[\begin{array}{cccccc}
-X+Y & 0 & \Phi_{1} \bar{V} & 0 & 0 & 0 \\
0 & -X-Y & 0 & \Phi_{2}^{1} \bar{Z} & 0 & 0 \\
\bar{V}^{T} \Phi_{1}^{T} & 0 & -\bar{V}-\bar{V}^{T} & 0 & X & 0 \\
0 & \bar{Z}^{T} \Phi_{2}^{1 T} & 0 & -\bar{Z}-\bar{Z}^{T} & \Phi_{2}^{2} \bar{V} & \Phi_{2}^{2} \bar{V} \\
0 & 0 & X & \bar{V}^{T} \Phi_{2}^{2 T} & -X & 0 \\
0 & 0 & 0 & \bar{V}^{T} \Phi_{2}^{2 T} & 0 & X-\bar{V}-\bar{V}^{T}
\end{array}\right]<0
$$

Finally, use of

$$
\begin{aligned}
& \Phi_{1} \bar{V}=\bar{A}_{1} \bar{V}+\bar{B}_{1} \bar{K}_{1} \bar{V}=\bar{A}_{1} \bar{V}+\bar{B}_{1} \bar{L}, \\
& \Phi_{2}^{1} \bar{Z}=\bar{A}_{2}^{1} \bar{Z}+\bar{B}_{2}^{1} \bar{K}_{2} \bar{Z}=\bar{A}_{2}^{1} \bar{Z}+\bar{B}_{2}^{1} \bar{N}, \\
& \Phi_{2}^{2} \bar{V}=\bar{A}_{2}^{2} \bar{V}+\bar{B}_{2}^{2} \bar{K}_{1} \bar{V}=\bar{A}_{2}^{2} \bar{V}+\bar{B}_{2}^{2} \bar{L}
\end{aligned}
$$


leads to (9) with stabilizing control law matrices given by (10) and the proof is complete.

It is of interest also to consider a simplified version of the control law developed above. This arises when $K_{2}^{2}=0$, i.e., the pass profile contribution to the control law is only applied on even passes. If it is possible to obtain stability along the pass in this case then a reduction in complexity of the overall approach will be achieved. The following is the major result in this case.

Theorem 6 Suppose that a control law with the structure of (6) with $K_{2}^{2}=0$ is applied to a discrete linear repetitive process which can be written in the form (3). Then the resulting process is stable along the pass, if $\exists$ matrices $Y=\left[\begin{array}{ccc}Y_{1} & 0 & 0 \\ 0 & Y_{2} & 0 \\ 0 & 0 & Y_{3}\end{array}\right]>0, Z>0$, and $N=\left[\begin{array}{ccc}N_{1} & 0 & N_{3} \\ 0 & N_{2} & 0\end{array}\right]$ such that the following LMI holds

$\left[\begin{array}{ccc}Z-Y & 0 & Y \widehat{A}_{1}^{T}+N^{T} \widehat{B}_{1}^{T} \\ 0 & -Z & Y \widehat{A}_{2}^{T}+N^{T} \widehat{B}_{2}^{T} \\ \widehat{A}_{1} Y+\widehat{B}_{1} N & \widehat{A}_{2} Y+\widehat{B}_{2} N & -Y\end{array}\right]<0$

where $\widehat{A}_{1}$, and $\widehat{A}_{2}$ are given by (4) and

$\widehat{B}_{1}=\left[\begin{array}{c}\widehat{B} \\ 0\end{array}\right]=\left[\begin{array}{cc}B_{1} & 0 \\ B_{02} D_{1} & B_{2} \\ 0 & 0\end{array}\right], \quad \widehat{B}_{2}=\left[\begin{array}{l}0 \\ \widehat{D}\end{array}\right]=\left[\begin{array}{cc}0 & 0 \\ 0 & 0 \\ D_{02} D_{1} & D_{2}\end{array}\right]$

If this condition holds, the control law matrices are given by

$\left[\begin{array}{ccc}K_{1}^{1} & 0 & K_{2}^{1} \\ 0 & K_{1}^{2} & 0\end{array}\right]=N Y^{-1}, \quad K_{2}^{2}=0$

Proof Follows immediately on applying the LMI based result of Theorem 3 to the controlled process state-space model in this case.

Note that this last result becomes increasingly conservative as the number of switching instants increases.

Next we give an illustrative example.

Example 1 Consider the special case of (2) when

$$
\begin{aligned}
& A_{1}=\left[\begin{array}{ccc}
0.60 & 0.20 & 0.10 \\
-0.30 & 0.40 & 2.00 \\
2.00 & -0.50 & 4.00
\end{array}\right], \quad B_{1}=\left[\begin{array}{cc}
0.30 & 0.70 \\
0.40 & 0.60 \\
-0.80 & 0.70
\end{array}\right], \quad B_{01}=\left[\begin{array}{cc}
-0.60 & 0.70 \\
-0.20 & 1.00 \\
0.10 & 0.10
\end{array}\right] \\
& C_{1}=\left[\begin{array}{ccc}
2.00 & -0.50 & -1.00 \\
-1.00 & 2.00 & -0.90
\end{array}\right], \quad D_{1}=\left[\begin{array}{cc}
-1.00 & -0.60 \\
1.00 & -0.10
\end{array}\right], \quad D_{01}=\left[\begin{array}{cc}
1.00 & -2.00 \\
-0.30 & 0.10
\end{array}\right]
\end{aligned}
$$

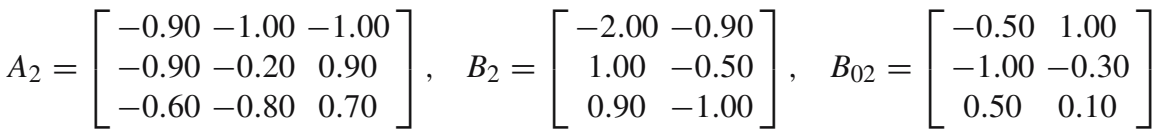

$$
\begin{aligned}
& C_{2}=\left[\begin{array}{ccc}
-0.60 & -2.00 & -2.00 \\
0.30 & 1.00 & -0.30
\end{array}\right], \quad D_{2}=\left[\begin{array}{cc}
-1.00 & -3.00 \\
-0.10 & 0.90
\end{array}\right], \quad D_{02}=\left[\begin{array}{c}
-1.00-1.00 \\
-2.00-1.00
\end{array}\right]
\end{aligned}
$$


which (as easily checked) is neither asymptotically or practically stable. The LMI stabilization condition of Theorem 5 holds in this case with

$$
\begin{aligned}
X & =\left[\begin{array}{c|c}
X_{1} & 0 \\
\hline 0 & X_{2}
\end{array}\right] \\
& =\left[\begin{array}{cccccc|cc}
0.9912 & -1.0807 & 0.4008 & 0 & 0 & 0 & 0 & 0 \\
-1.0807 & 1.2624 & -0.4268 & 0 & 0 & 0 & 0 & 0 \\
0.4008 & -0.4268 & 0.2003 & 0 & 0 & 0 & 0 & 0 \\
0 & 0 & 0 & 1.4569 & 0.5031 & 0.5200 & 0 & 0 \\
0 & 0 & 0 & 0.5031 & 2.6059 & 0.8290 & 0 & 0 \\
0 & 0 & 0 & 0.5200 & 0.8290 & 1.3534 & 0 & 0 \\
\hline 0 & 0 & 0 & 0 & 0 & 0 & 4.5599 & 1.7188 \\
0 & 0 & 0 & 0 & 0 & 0 & 1.7188 & 0.7156
\end{array}\right] \\
Y & {\left[\begin{array}{cccccccc}
0.2004 & -0.2320 & 0.0923 & 0 & 0 & 0 & 0 & 0 \\
-0.2320 & 0.2934 & -0.1020 & 0 & 0 & 0 & 0 & 0 \\
0.0923 & -0.1020 & 0.0604 & 0 & 0 & 0 & 0 & 0 \\
0 & 0 & 0 & 0.5877 & 0.0091 & 0.1044 & 0.1041 & 0.0400 \\
0 & 0 & 0 & 0.0091 & 0.9607 & 0.2193 & 0.4394 & 0.1734 \\
0 & 0 & 0 & 0.1044 & 0.2193 & 0.5618 & 0.2983 & 0.1171 \\
0 & 0 & 0 & 0.1041 & 0.4394 & 0.2983 & 2.4565 & 0.9568 \\
0 & 0 & 0 & 0.0400 & 0.1734 & 0.1171 & 0.9568 & 0.4118
\end{array}\right] }
\end{aligned}
$$$$
\begin{aligned}
\bar{V} & =\left[\begin{array}{c|c|c}
V_{1} & 0 & 0 \\
\hline 0 & V_{2} & 0 \\
\hline 0 & 0 & V_{3}
\end{array}\right] \\
& =\left[\begin{array}{ccc|ccc|cc}
0.8451 & -0.9172 & 0.3416 & 0 & 0 & 0 & 0 & 0 \\
-0.9371 & 1.0960 & -0.3690 & 0 & 0 & 0 & 0 & 0 \\
0.3534 & -0.3752 & 0.1832 & 0 & 0 & 0 & 0 & 0 \\
\hline 0 & 0 & 0 & 1.4676 & 0.2440 & 0.2827 & 0 & 0 \\
0 & 0 & 0 & 0.2189 & 2.1050 & 0.3211 & 0 & 0 \\
0 & 0 & 0 & 0.4198 & 0.6512 & 1.3797 & 0 & 0 \\
\hline 0 & 0 & 0 & 0 & 0 & 0 & 3.3049 & 1.2759 \\
0 & 0 & 0 & 0 & 0 & 0 & 1.1981 & 0.5315
\end{array}\right]
\end{aligned}
$$$$
\bar{Z}=\left[\begin{array}{c|c|c}
Z & 0 & 0 \\
\hline 0 & Z & 0 \\
\hline 0 & 0 & Z
\end{array}\right], \quad Z=\left[\begin{array}{cc}
0.9192 & -0.6435 \\
-0.5964 & 1.8055
\end{array}\right]
$$$$
\bar{L}=\left[\begin{array}{c|c|c}
L_{1} & 0 & 0 \\
\hline 0 & L_{2} & 0 \\
\hline 0 & 0 & L_{3}
\end{array}\right]
$$$$
=\left[\begin{array}{ccc|ccc|cc}
2.8775 & -3.1546 & 1.2632 & 0 & 0 & 0 & 0 & 0 \\
-1.8642 & 2.0029 & -0.8821 & 0 & 0 & 0 & 0 & 0 \\
\hline 0 & 0 & 0 & -0.4080 & -0.6478 & -0.8621 & 0 & 0 \\
0 & 0 & 0 & -0.6101 & -1.9368 & -0.5422 & 0 & 0 \\
\hline 0 & 0 & 0 & 0 & 0 & 0 & 1.0025 & 0.3391 \\
0 & 0 & 0 & 0 & 0 & 0 & 0.0270 & -0.0694
\end{array}\right]
$$ 
$\bar{N}=\left[\begin{array}{c|c|c}N & 0 & 0 \\ \hline 0 & N & 0 \\ \hline 0 & 0 & N\end{array}\right], \quad N=\left[\begin{array}{cc}-0.5354 & 0.5605 \\ 0.5863 & -0.0323\end{array}\right]$

The eigenvalues of the matrices $X$ and $Y$ are

$\operatorname{eig}(X)=\left[\begin{array}{l}0.0225 \\ 0.0578 \\ 0.0592 \\ 0.8080 \\ 1.3306 \\ 2.3736 \\ 3.2777 \\ 5.2163\end{array}\right], \quad \operatorname{eig}(Y)=\left[\begin{array}{l}0.0069 \\ 0.0230 \\ 0.0339 \\ 0.4131 \\ 0.5242 \\ 0.6268 \\ 0.8974 \\ 3.0072\end{array}\right]$

and hence they are positive definite.

The only remaining potential difficulty numerically is associated with constructing the inverses of the square matrices $\bar{V}$ and $\bar{Z}$ to compute the stabilizing control law matrices. The condition numbers of these matrices are given by

$\operatorname{cond}(\bar{V})=166.4848, \quad \operatorname{cond}(\bar{Z})=3.5382$

and hence constructing these matrix inverses to give the control law matrices is not a problem and we have

$$
\begin{aligned}
& \bar{K}_{1}=\bar{L} \bar{V}^{-1}=\left[\begin{array}{c|c|c}
K_{1}^{1} & 0 & 0 \\
\hline 0 & K_{1}^{2} & 0 \\
\hline 0 & 0 & K_{2}^{1}
\end{array}\right] \\
& =\left[\begin{array}{ccc|ccc|cc}
1.5485 & -0.6781 & 2.6410 & 0 & 0 & 0 & 0 & 0 \\
-0.7767 & 0.0807 & -3.2035 & 0 & 0 & 0 & 0 & 0 \\
\hline 0 & 0 & 0 & -0.0951 & -0.1179 & -0.5779 & 0 & 0 \\
0 & 0 & 0 & -0.2479 & -0.8464 & -0.1452 & 0 & 0 \\
\hline 0 & 0 & 0 & 0 & 0 & 0 & 0.5553 & -0.6952 \\
0 & 0 & 0 & 0 & 0 & 0 & 0.4276 & -1.1570
\end{array}\right] \\
& \bar{K}_{2}=\bar{N} \bar{Z}^{-1}=\left[\begin{array}{c|c|c}
K_{2}^{2} & 0 & 0 \\
\hline 0 & K_{2}^{2} & 0 \\
\hline 0 & 0 & K_{2}^{2}
\end{array}\right], \quad K_{2}^{2}=\left[\begin{array}{cc}
-0.4957 & 0.1338 \\
0.8146 & 0.2724
\end{array}\right]
\end{aligned}
$$

The controlled process is described by the following set of state-space matrices

$$
\begin{array}{ll}
A_{1 \text { new }}=A_{1}+B_{1} K_{1}^{1}, & B_{01 \text { new }}=B_{01}+B_{1} K_{2}^{1} \\
A_{2 \text { new }}=A_{2}+B_{2} K_{1}^{2}, & B_{02 \text { new }}=B_{02}+B_{2} K_{2}^{2} \\
C_{1 \text { new }}=C_{1}+D_{1} K_{1}^{1}, & D_{01 \text { new }}=D_{01}+D_{1} K_{2}^{1} \\
C_{\text {2new }}=C_{2}+D_{2} K_{1}^{2}, & D_{02 \text { new }}=D_{02}+D_{2} K_{2}^{2}
\end{array}
$$




$$
\begin{aligned}
& A_{1 \text { new }}=\left[\begin{array}{ccc}
0.5208 & 0.0530 & -1.3502 \\
-0.1466 & 0.1772 & 1.1343 \\
0.2174 & 0.0990 & -0.3553
\end{array}\right], \quad B_{01 \text { new }}=\left[\begin{array}{cc}
-0.1341 & -0.3184 \\
0.2787 & 0.0277 \\
-0.0450 & -0.1537
\end{array}\right] \\
& A_{2 \text { new }}=\left[\begin{array}{ccc}
-0.4866 & -0.0024 & 0.2865 \\
-0.8712 & 0.1053 & 0.3947 \\
-0.4377 & -0.0597 & 0.3251
\end{array}\right], \quad B_{02 \text { new }}=\left[\begin{array}{cc}
-0.2418 & 0.4872 \\
-1.9030 & -0.3024 \\
-0.7607 & -0.0520
\end{array}\right] \\
& C_{1 \text { new }}=\left[\begin{array}{lll}
0.9175 & 0.1297 & -1.7189 \\
0.6262 & 1.3138 & 2.0614
\end{array}\right], \quad D_{01 \text { new }}=\left[\begin{array}{l}
0.1881-0.6106 \\
0.2126-0.4795
\end{array}\right] \\
& C_{\text {2new }}=\left[\begin{array}{lll}
0.2389 & 0.6572 & -0.9865 \\
0.0864 & 0.2500 & -0.3729
\end{array}\right], \quad D_{02 \text { new }}=\left[\begin{array}{l}
-2.9481-1.9511 \\
-1.2173-0.7682
\end{array}\right]
\end{aligned}
$$

\section{Discrete linear repetitive processes with dynamics switched along the pass}

We can extend the analysis of the previous section to the following model of the case when the repetitive process dynamics are subject to switching along the pass as defined by

$$
\begin{gathered}
x_{k+1}(s+1)=\left\{\begin{array}{l}
A_{1} x_{k+1}(s)+B_{1} u_{k+1}(s)+B_{01} y_{k}(s), \text { for } s=0,2, \ldots, \text { i.e., } s=2 p \\
A_{2} x_{k+1}(s)+B_{2} u_{k+1}(s)+B_{02} y_{k}(s), \text { for } s=1,3, \ldots, \text { i.e., } s=2 p+1
\end{array}\right. \\
y_{l+1}(s)=\left\{\begin{array}{l}
C_{1} x_{k+1}(s)+D_{1} u_{k+1}(s)+D_{01} y_{k}(s), \text { for } s=0,2, \ldots, \text { i.e., } s=2 p \\
C_{2} x_{k+1}(s)+D_{2} u_{k+1}(s)+D_{02} y_{k}(s), \text { for } s=1,3, \ldots, \text { i.e., } s=2 p+1
\end{array}\right.
\end{gathered}
$$

and (excluding $s$ ) all variables have the meanings assigned to them in the previous section.

The dynamics described by (11) can be written in the from of (1) as

$$
\begin{aligned}
X_{k+1}(s+1) & =\check{A} X_{k+1}(s)+\check{B} U_{k+1}(s)+\check{B}_{0} Y_{k}(s) \\
Y_{k+1}(s) & =\check{C} X_{k+1}(s)+\check{D} U_{k+1}(s)+\check{D}_{0} Y_{k}(s)
\end{aligned}
$$

where

$$
\begin{aligned}
& X_{k+1}(s)=x_{k+1}(2 p), \quad X_{k+1}(s+1)=x_{k+1}(2 p+2), \quad U_{k+1}(s)=\left[\begin{array}{c}
u_{k+1}(2 p) \\
u_{k+1}(2 p+1)
\end{array}\right] \\
& Y_{k}(s)=\left[\begin{array}{c}
y_{k}(2 p) \\
y_{k}(2 p+1)
\end{array}\right]
\end{aligned}
$$

and

$$
\begin{array}{lll}
\check{A}=A_{2} A_{1}, & \check{B}=\left[\begin{array}{cc}
A_{2} B_{1} & B_{2}
\end{array}\right], & \check{B}_{0}=\left[\begin{array}{cc}
A_{2} B_{01} & B_{02}
\end{array}\right] \\
\check{C}=\left[\begin{array}{c}
C_{1} \\
C_{2} A_{1}
\end{array}\right], & \check{D}=\left[\begin{array}{cc}
D_{1} & 0 \\
C_{2} B_{1} & D_{2}
\end{array}\right], & \check{D}_{0}=\left[\begin{array}{cc}
D_{01} & 0 \\
C_{2} B_{01} & D_{02}
\end{array}\right]
\end{array}
$$

This model comes directly from lifting the models which switch along the pass, producing an equivalent description which is parameter invariant but with doubled dimensions. As noted previously, this approach has been used in iterative learning control, see, for example, (De Roover et al., 2000; Moore, 2000; Norrlof \& Gunnarsson, 2002; Rotariu et al., 2004) but most often the end point in analysis is to produce an equivalent $1 D$ model for stability analysis over the finite trial length, i.e., asymptotic stability. In the analysis here it is the stronger property of stability along the pass which is considered and we aim to design the control law using the basic (i.e., non-lifted) model. This will especially important when considering the more general case when there is uncertainty associated with the process description. In 
particular, the only feasible approach to analysis in such a case is to assume an uncertainty description and this will not be preserved if a lifted model is used for analysis.

If we are only interested in asymptotic stability or practical stability then the following result is immediate from application of the conditions for these properties to hold for processes considered in the previous section.

Theorem 7 A discrete linear repetitive process which can be written in the form (12) is asymptotically stable if, and only if, $r\left(D_{01}\right)<1$ and $r\left(D_{02}\right)<1$ and practically stable if, and only if, asymptotic stability holds and $r\left(A_{2} A_{1}\right)<1$.

Proof Follows immediately on applying the respective stability conditions to the lifted model (12).

For the stability along the pass we obtain the following result by interpreting Corollary 1 in terms of (12).

Theorem 8 A discrete linear repetitive process which can be written in the form (12) is stable along the pass if $\exists$ matrices $W_{1}>0$ and $W_{2}>0$ such that

$\check{\Phi}^{T} W \check{\Phi}-W<0$

holds where here the augmented plant matrix $\check{\Phi}$ is given by

$\check{\Phi}=\left[\begin{array}{cc}\check{A} & \check{B_{0}} \\ \check{C} & \check{D_{0}}\end{array}\right]$

and (as in the analysis of the previous section) $W=W_{1} \oplus W_{2}$.

As a first attempt at control of these processes we consider a switched control law of the form

$u_{k+1}(s)=\left\{\begin{array}{l}K_{1}^{1} x_{k+1}(s)+K_{2}^{1} y_{k}(s), \text { for } s=0,2, \ldots, \text { i.e., } s=2 p \\ K_{1}^{2} x_{k+1}(s)+K_{2}^{2} y_{k}(s), \text { for } s=1,3, \ldots, \text { i.e., } s=2 p+1\end{array}\right.$

or

$U_{k+1}(s)=\check{K}_{1} X_{k+1}(s)+\check{K}_{2} Y_{k}(s)$

where

$\check{K}_{1}=\left[\begin{array}{c}K_{1}^{1} \\ K_{1}^{2}\left(A_{1}+B_{1} K_{1}^{1}\right)\end{array}\right], \quad \check{K}_{2}=\left[\begin{array}{cc}K_{2}^{1} & 0 \\ K_{1}^{2}\left(B_{01}+B_{1} K_{2}^{1}\right) & K_{2}^{2}\end{array}\right]$

The resulting controlled process state-space model can be written as

$$
\begin{aligned}
X_{k+1}(s+1) & =\check{A}_{\text {new }} X_{k+1}(s)+\check{B}_{\text {0new }} Y_{k}(s) \\
Y_{k+1}(s) & =\check{C}_{\text {new }} X_{k+1}(s)+\check{D}_{\text {Onew }} Y_{k}(s)
\end{aligned}
$$

where

$$
\begin{aligned}
& \check{A}_{\text {new }}=\check{A}+\check{B} \check{K}_{1}=\left[\left(A_{2}+B_{2} K_{1}^{2}\right)\left(A_{1}+B_{1} K_{1}^{1}\right)\right], \\
& \check{B}_{\text {new }}=\check{B}_{0}+\check{B} \check{K}_{2}=\left[\left(A_{2}+B_{2} K_{1}^{2}\right)\left(B_{01}+B_{1} K_{2}^{1}\right) B_{02}+B_{2} K_{2}^{2}\right], \\
& \check{C}_{\text {new }}=\check{C}+\check{D}_{1}=\left[\begin{array}{c}
C_{1}+D_{1} K_{1}^{1} \\
\left(C_{2}+D_{2} K_{1}^{2}\right)\left(A_{1}+B_{1} K_{1}^{1}\right)
\end{array}\right], \\
& \check{D}_{\text {0new }}=\check{D}_{0}+\check{D}_{2}=\left[\begin{array}{cc}
D_{01}+D_{1} K_{2}^{1} & 0 \\
\left(C_{2}+D_{2} K_{1}^{2}\right)\left(B_{01}+B_{1} K_{2}^{1}\right) D_{02}+D_{2} K_{2}^{2}
\end{array}\right]
\end{aligned}
$$


Also introduce

$\check{\Phi}_{\text {new }}=\left[\begin{array}{cc}\check{A}_{\text {new }} & \check{B}_{0 \text { new }} \\ \check{C}_{\text {new }} & \check{D}_{\text {Onew }}\end{array}\right]=\left[\begin{array}{cc}\check{A} & \check{B}_{0} \\ \check{C} & \check{D}_{0}\end{array}\right]+\left[\begin{array}{c}\check{B} \\ \check{D}\end{array}\right]\left[\begin{array}{ll}\check{K}_{1} & \check{K}_{2}\end{array}\right]$

and hence we can write

$\check{\Phi}_{\text {new }}=\left[\begin{array}{ccc}\left(A_{2}+B_{2} K_{1}^{2}\right)\left(A_{1}+B_{1} K_{1}^{1}\right) & \left(A_{2}+B_{2} K_{1}^{2}\right)\left(B_{01}+B_{1} K_{2}^{1}\right) & B_{02}+B_{2} K_{2}^{2} \\ C_{1}+D_{1} K_{1}^{1} & D_{01}+D_{1} K_{2}^{1} & 0 \\ \left(C_{2}+D_{2} K_{1}^{2}\right)\left(A_{1}+B_{1} K_{1}^{1}\right) & \left(C_{2}+D_{2} K_{1}^{2}\right)\left(B_{01}+B_{1} K_{2}^{1}\right) & D_{02}+D_{2} K_{2}^{2}\end{array}\right]$

or, equivalently,

$\check{\Phi}_{\text {new }}=\Phi_{1}+\Phi_{2}^{1} \Phi_{2}^{2}$

where

$\Phi_{1}=\widetilde{A}_{1}+\widetilde{B}_{1} \widetilde{K}_{1}, \quad \Phi_{2}^{1}=\widetilde{A}_{2}^{1}+\widetilde{B}_{2}^{1} \widetilde{K}_{2}, \quad \Phi_{2}^{2}=\widetilde{A}_{2}^{2}+\widetilde{B}_{2}^{2} \widetilde{K}_{1}$

and

$\widetilde{A}_{1}=\left[\begin{array}{ccc}0 & 0 & B_{02} \\ C_{1} & D_{01} & 0 \\ 0 & 0 & D_{02}\end{array}\right], \quad \widetilde{B}_{1}=\left[\begin{array}{ccc}0 & 0 & B_{2} \\ D_{1} & D_{1} & 0 \\ 0 & 0 & D_{2}\end{array}\right], \quad \widetilde{K}_{1}=\left[\begin{array}{ccc}K_{1}^{1} & 0 & 0 \\ 0 & K_{2}^{1} & 0 \\ 0 & 0 & K_{2}^{2}\end{array}\right]$

$\widetilde{A}_{2}^{1}=\left[\begin{array}{ccc}A_{2} & 0 & 0 \\ 0 & 0 & 0 \\ 0 & 0 & C_{2}\end{array}\right], \quad \widetilde{B}_{2}^{1}=\left[\begin{array}{ccc}B_{2} & 0 & 0 \\ 0 & 0 & 0 \\ 0 & 0 & D_{2}\end{array}\right], \quad \widetilde{K}_{2}=\left[\begin{array}{ccc}K_{1}^{2} & 0 & 0 \\ 0 & K_{1}^{2} & 0 \\ 0 & 0 & K_{1}^{2}\end{array}\right]$

$\widetilde{A}_{2}^{2}=\left[\begin{array}{ccc}A_{1} & B_{01} & 0 \\ 0 & 0 & 0 \\ A_{1} & B_{01} & 0\end{array}\right], \quad \widetilde{B}_{2}^{2}=\left[\begin{array}{ccc}B_{1} & B_{1} & 0 \\ 0 & 0 & 0 \\ B_{1} & B_{1} & 0\end{array}\right]$

4.1. Stabilization to achieve asymptotic and practical stability

The following result is the counterpart of Theorem 4 and is proved in an identical manner and hence the details are omitted here.

Theorem 9 Suppose that a control law with the structure of (13) is applied to a discrete linear repetitive process which can be written in the form (12). Then the resulting process is asymptotically stable if, and only if, $\exists$ matrices $X_{i}$, nonsingular matrices $V_{i}$ and matrices $M_{i}, i=1,2$ such that the following LMIs hold

$\left[\begin{array}{cc}-X_{i} & D_{0 i} V_{i}+D_{i} M_{i} \\ \left(D_{0 i} V_{i}+D_{i} M_{i}\right)^{T} & X_{i}-V_{i}-V_{i}^{T}\end{array}\right]<0, \quad i=1,2$

If these conditions hold then the control law matrices are given by

$K_{2}^{i}=M_{i} V_{i}^{-1}, \quad i=1,2$

and $K_{1}^{1}=K_{1}^{2}=0$. Also practical stability holds if, and only if, the last two LMIs hold and $\exists$ a matrix $X>0$, two nonsingular matrices $V$ and $G$ and two matrices $N_{1}$ and $N_{2}$ such that the following LMI holds

$\left[\begin{array}{cccc}-X & A_{2} V+B_{2} N_{2} & 0 & 0 \\ \left(A_{2} V+B_{2} N_{2}\right)^{T} & -V-V^{T} & 0 & A_{1} G+B_{1} N_{1} \\ 0 & 0 & -X & X \\ 0 & \left(A_{1} G+B_{1} N_{1}\right)^{T} & X^{T} & -G-G^{T}\end{array}\right]<0$

If this condition holds, the corresponding control law matrices are given by

$K_{1}^{1}=N_{1} G^{-1}, \quad K_{1}^{2}=N_{2} V^{-1}$

and $K_{2}^{i}, i=1,2$ are as in the asymptotic stability case.

悬 Springer 
4.2. Stabilization to achieve stability along the pass

Theorem 10 Suppose that a control law with the structure of (13) is applied to a discrete linear repetitive process which can be written in the form (12). Then the resulting process is stable along the pass, if $\exists$ matrices $X_{i}, i=1,2, Y$, square matrices $V_{i}, i=1,2,3$ and $Z$, and rectangular matrices $L_{i}, i=1,2,3$ and $N$ such that

$$
\begin{gathered}
{\left[\begin{array}{ccc}
-X+Y & 0 & \widetilde{A}_{1} \widetilde{V}+\widetilde{B}_{1} \widetilde{L} \\
0 & -X-Y & 0 \\
\widetilde{V}^{T} \widetilde{A}_{1}^{T}+\widetilde{L}^{T} \widetilde{B}_{1}^{T} & 0 & -\widetilde{V}-\widetilde{V}^{T} \\
0 & \widetilde{Z}^{T} \widetilde{A}_{2}^{1 T}+\widetilde{N}^{T} \widetilde{B}_{2}^{1 T} & 0 \\
0 & 0 & X \\
0 & 0 & 0 \\
& & \\
0 & 0 & 0 \\
\widetilde{A}_{2}^{1} \widetilde{Z}+\widetilde{B}_{2}^{1} \widetilde{N} & 0 & 0 \\
0 & X & 0 \\
-\widetilde{Z}-\widetilde{Z}^{T} & \widetilde{A}_{2}^{2} \widetilde{V}+\widetilde{B}_{2}^{2} \widetilde{L} \widetilde{A}_{2}^{2} \widetilde{V}+\widetilde{B}_{2}^{2} \widetilde{L} \\
\widetilde{V}^{T} \widetilde{A}_{2}^{2 T}+\widetilde{L}^{T} \widetilde{B}_{2}^{2 T} & -X & 0 \\
\widetilde{V}^{T} \widetilde{A}_{2}^{2 T}+\widetilde{L}^{T} \widetilde{B}_{2}^{2 T} & 0 & X-\widetilde{V}-\widetilde{V}^{T}
\end{array}\right]<0}
\end{gathered}
$$

where

$$
\begin{aligned}
& X=\left[\begin{array}{cc}
X_{1} & 0 \\
0 & X_{2}
\end{array}\right], \quad \widetilde{V}=\left[\begin{array}{ccc}
V_{1} & 0 & 0 \\
0 & V_{2} & 0 \\
0 & 0 & V_{3}
\end{array}\right], \quad \widetilde{Z}=\left[\begin{array}{lll}
Z & 0 & 0 \\
0 & Z & 0 \\
0 & 0 & Z
\end{array}\right] \\
& \widetilde{L}=\left[\begin{array}{ccc}
L_{1} & 0 & 0 \\
0 & L_{2} & 0 \\
0 & 0 & L_{3}
\end{array}\right], \quad \widetilde{N}=\left[\begin{array}{ccc}
N & 0 & 0 \\
0 & N & 0 \\
0 & 0 & N
\end{array}\right]
\end{aligned}
$$

If this condition holds, the control law matrices are given by

$\widetilde{K}_{1}=\widetilde{L} \widetilde{V}^{-1}=\left[\begin{array}{ccc}K_{1}^{1} & 0 & 0 \\ 0 & K_{2}^{1} & 0 \\ 0 & 0 & K_{2}^{2}\end{array}\right], \quad \widetilde{K}_{2}=\widetilde{N} \widetilde{Z}^{-1}=\left[\begin{array}{ccc}K_{1}^{2} & 0 & 0 \\ 0 & K_{1}^{2} & 0 \\ 0 & 0 & K_{1}^{2}\end{array}\right]$

Proof This follows identical steps to that of Theorem 5 and hence the details are omitted here.

The following result is the counterpart of Theorem 6 of the previous section.

Theorem 11 Suppose that a control law of the form (13) with $K_{1}^{2}=0$ is applied to a discrete linear repetitive process which can be written in the form (12). Then the resulting process is stable along the pass, if $\exists$ matrices $\quad Y=\left[\begin{array}{ccc}Y_{1} & 0 & 0 \\ 0 & Y_{2} & 0 \\ 0 & 0 & Y_{3}\end{array}\right]>0, Z>0$, and $N=\left[\begin{array}{ccc}N_{1} & N_{2} & 0 \\ 0 & 0 & N_{3}\end{array}\right]$ such that

$$
\left[\begin{array}{ccc}
Z-Y & 0 & Y \check{A}_{1}^{T}+N^{T} \check{B}_{1}^{T} \\
0 & -Z & Y \check{A}_{2}^{T}+N^{T} \check{B}_{2}^{T} \\
\check{A}_{1} Y+\check{B}_{1} N & \check{A}_{2} Y+\check{B}_{2} N & -Y
\end{array}\right]<0
$$

where

$$
\check{A}_{1}=\left[\begin{array}{cc}
\check{A} & \check{B}_{0} \\
0 & 0
\end{array}\right], \check{A}_{2}=\left[\begin{array}{cc}
0 & 0 \\
\check{C} & \check{D}_{0}
\end{array}\right], \quad \check{B}_{1}=\left[\begin{array}{c}
\check{B} \\
0
\end{array}\right], \check{B}_{2}=\left[\begin{array}{l}
0 \\
\check{D}
\end{array}\right]
$$


If this condition holds, the matrices in the stabilizing control law are given by

$\left[\begin{array}{ccc}K_{1}^{1} & K_{2}^{1} & 0 \\ 0 & 0 & K_{2}^{2}\end{array}\right]=N Y^{-1}, \quad K_{1}^{2}=0$

Proof This is a straightforward application of known LMI results for discrete linear repetitive processes of the form (1) (given in (Galkowski et al., 2002)) and hence the details are omitted here.

Next we give an illustrative example.

Example 2 Consider the case when

$$
\begin{aligned}
& A_{1}=\left[\begin{array}{ccc}
0.60 & 0.20 & 0.10 \\
-0.30 & 0.40 & 2.00 \\
2.00 & -0.50 & 4.00
\end{array}\right], \quad B_{1}=\left[\begin{array}{cc}
0.30 & 0.70 \\
0.40 & 0.60 \\
-0.80 & 0.70
\end{array}\right], \quad B_{01}=\left[\begin{array}{cc}
-0.60 & 0.70 \\
-0.20 & 1.00 \\
0.10 & 0.10
\end{array}\right] \\
& C_{1}=\left[\begin{array}{ccc}
2.00 & -0.50 & -1.00 \\
-1.00 & 2.00 & -0.90
\end{array}\right], \quad D_{1}=\left[\begin{array}{cc}
-1.00 & -0.60 \\
1.00 & -0.10
\end{array}\right], \quad D_{01}=\left[\begin{array}{cc}
1.00 & -2.00 \\
-0.30 & 0.10
\end{array}\right]
\end{aligned}
$$

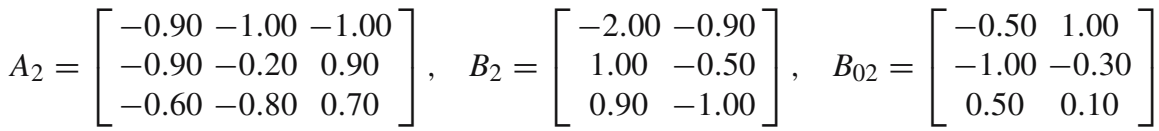

$$
\begin{aligned}
& C_{2}=\left[\begin{array}{ccc}
-0.60 & -2.00 & -2.00 \\
0.30 & 1.00 & -0.30
\end{array}\right], \quad D_{2}=\left[\begin{array}{cc}
-1.00 & -3.00 \\
-0.10 & 0.90
\end{array}\right], \quad D_{02}=\left[\begin{array}{c}
-1.00-1.00 \\
-2.00-1.00
\end{array}\right]
\end{aligned}
$$

which (as easily checked) is neither asymptotically or practically stable. The LMI stabilization condition of Theorem 10 is feasible in this case with

$$
\begin{aligned}
X & =\left[\begin{array}{c|c}
X_{1} & 0 \\
\hline 0 & X_{2}
\end{array}\right] \\
& =\left[\begin{array}{ccc|cccc}
135.9258 & -68.4758 & 40.7509 & 0 & 0 & 0 & 0 \\
-68.4758 & 135.9616 & -27.4434 & 0 & 0 & 0 & 0 \\
40.7509 & -27.4434 & 16.8076 & 0 & 0 & 0 & 0 \\
\hline 0 & 0 & 0 & 349.4949 & 144.8589 & 0 & 0 \\
0 & 0 & 0 & 144.8589 & 248.3737 & 0 & 0 \\
0 & 0 & 0 & 0 & 0 & 17.3710 & -24.5412 \\
0 & 0 & 0 & 0 & 0 & -24.5412 & 42.3985
\end{array}\right]
\end{aligned}
$$

$$
Y=\left[\begin{array}{ccccccc}
39.0914 & -41.7387 & 12.6522 & 0 & 0 & -3.0846 & 6.5937 \\
-41.7387 & 57.3999 & -14.8988 & 0 & 0 & 1.0618 & -1.1344 \\
12.6522 & -14.8988 & 6.6037 & 0 & 0 & -0.9977 & 2.0390 \\
0 & 0 & 0 & 27.0446 & -4.9498 & 0 & 0 \\
0 & 0 & 0 & -4.9498 & 7.1693 & 0 & 0 \\
-3.0846 & 1.0618 & -0.9977 & 0 & 0 & 7.5033 & -9.5228 \\
6.5937 & -1.1344 & 2.0390 & 0 & 0 & -9.5228 & 15.9507
\end{array}\right]
$$




$$
\begin{aligned}
\widetilde{V} & =\left[\begin{array}{c|c|c}
V_{1} & 0 & 0 \\
\hline 0 & V_{2} & 0 \\
\hline 0 & 0 & V_{3}
\end{array}\right] \\
& =\left[\begin{array}{ccc|cc|cc}
123.0823 & -53.6655 & 35.9548 & 0 & 0 & 0 & 0 \\
-57.3519 & 126.3152 & -23.3325 & 0 & 0 & 0 & 0 \\
36.2814 & -22.3292 & 15.3079 & 0 & 0 & 0 & 0 \\
\hline 0 & 0 & 0 & 273.8613 & 132.7959 & 0 & 0 \\
0 & 0 & 0 & 61.6835 & 235.4204 & 0 & 0 \\
\hline 0 & 0 & 0 & 0 & 0 & 19.4603 & -26.0767 \\
0 & 0 & 0 & 0 & 0 & -25.8828 & 44.8662
\end{array}\right]
\end{aligned}
$$

$\widetilde{Z}=\left[\begin{array}{c|c|c}Z & 0 & 0 \\ \hline 0 & Z & 0 \\ \hline 0 & 0 & Z\end{array}\right], \quad Z=\left[\begin{array}{ccc}126.7049 & -20.0403 & 73.0894 \\ -231.7577 & 341.2378 & -9.8435 \\ -19.1441 & 117.9366 & 68.4457\end{array}\right]$

$$
\begin{aligned}
\widetilde{L} & =\left[\begin{array}{c|c|c}
L_{1} & 0 & 0 \\
\hline 0 & L_{2} & 0 \\
\hline 0 & 0 & L_{3}
\end{array}\right] \\
& =\left[\begin{array}{ccc|cc|cc}
317.4386 & -232.0404 & 109.3331 & 0 & 0 & 0 & 0 \\
-224.3303 & 111.5687 & -75.3270 & 0 & 0 & 0 & 0 \\
\hline 0 & 0 & 0 & 91.0851 & -90.2622 & 0 & 0 \\
0 & 0 & 0 & 99.1580 & -135.9259 & 0 & 0 \\
\hline 0 & 0 & 0 & 0 & 0 & -9.0485 & 10.5439 \\
0 & 0 & 0 & 0 & 0 & 3.2294 & -9.9063
\end{array}\right]
\end{aligned}
$$

$$
\tilde{N}=\left[\begin{array}{c|c|c}
N & 0 & 0 \\
\hline 0 & N & 0 \\
\hline 0 & 0 & N
\end{array}\right], \quad N=\left[\begin{array}{ccc}
22.0190 & -94.8900 & -56.4713 \\
115.9953 & -256.2702 & -42.1948
\end{array}\right]
$$

The eigenvalues of the matrices $X$ and $Y$ are

$$
\operatorname{eig}(X)=\left[\begin{array}{c}
2.3373 \\
3.8234 \\
57.4323 \\
68.7514 \\
145.5052 \\
216.1201 \\
452.3634
\end{array}\right], \quad \operatorname{eig}(Y)=\left[\begin{array}{c}
1.0296 \\
2.2178 \\
4.5770 \\
6.0048 \\
22.9387 \\
28.2091 \\
95.7859
\end{array}\right]
$$

and hence they are positive definite. 
The only remaining potential difficulty numerically is associated with constructing the inverses of the matrices $\tilde{V}$ and $\tilde{Z}$ respectively to compute the stabilizing control laws. The condition numbers of the matrices are given by

$\operatorname{cond}(\widetilde{V})=109.8010, \quad \operatorname{cond}(\widetilde{Z})=21.6952$

and we have

$$
\begin{aligned}
& \widetilde{K}_{1}=\widetilde{L} \widetilde{V}^{-1}=\left[\begin{array}{c|c|c}
K_{1}^{1} & 0 & 0 \\
\hline 0 & K_{2}^{1} & 0 \\
\hline 0 & 0 & K_{2}^{2}
\end{array}\right] \\
& =\left[\begin{array}{ccc|cc|cc}
1.4983 & -0.7665 & 2.4547 & 0 & 0 & 0 & 0 \\
-1.2093 & 0.0024 & -2.0767 & 0 & 0 & 0 & 0 \\
\hline 0 & 0 & 0 & 0.4799 & -0.6541 & 0 & 0 \\
0 & 0 & 0 & 0.5637 & -0.8954 & 0 & 0 \\
\hline 0 & 0 & 0 & 0 & 0 & -0.6715-0.1553 \\
0 & 0 & 0 & 0 & 0 & -0.5627 & -0.5479
\end{array}\right] \\
& \widetilde{K}_{2}=\tilde{N} \widetilde{Z}^{-1}=\left[\begin{array}{c|c|c}
K_{1}^{2} & 0 & 0 \\
\hline 0 & K_{1}^{2} & 0 \\
\hline 0 & 0 & K_{1}^{2}
\end{array}\right], \quad K_{1}^{2}=\left[\begin{array}{ccc}
0.1513 & 0.0684 & -0.9768 \\
-0.3099 & -0.6388 & -0.3774
\end{array}\right]
\end{aligned}
$$

The resulting controlled process is characterized by the following state-space matrices

$$
\begin{aligned}
& A_{1 \text { new }}=\left[\begin{array}{ccc}
0.2030 & -0.0283 & -0.6173 \\
-0.4263 & 0.0948 & 1.7359 \\
-0.0452 & 0.1148 & 0.5825
\end{array}\right], \quad B_{01 \text { new }}=\left[\begin{array}{cc}
-0.0614 & -0.1230 \\
0.3302 & 0.2011 \\
0.1107 & -0.0035
\end{array}\right] \\
& A_{2 \text { new }}=\left[\begin{array}{ccc}
-0.9237 & -0.5619 & 1.2932 \\
-0.5937 & 0.1878 & 0.1119 \\
-0.1539 & -0.0997 & 0.1982
\end{array}\right], \quad B_{02 \text { new }}=\left[\begin{array}{cc}
1.3494 & 1.8036 \\
-1.3901 & -0.1813 \\
0.4584 & 0.5081
\end{array}\right] \\
& C_{1 \text { new }}=\left[\begin{array}{lll}
1.2273 & 0.2651 & -2.2087 \\
0.6193 & 1.2333 & 1.7624
\end{array}\right], \quad D_{01 \text { new }}=\left[\begin{array}{cc}
0.1818-0.8087 \\
0.1236-0.4646
\end{array}\right] \\
& C_{2 \text { new }}=\left[\begin{array}{lll}
0.1785 & -0.1521 & 0.1089 \\
0.0059 & 0.4183 & -0.5419
\end{array}\right], \quad D_{02 \text { new }}=\left[\begin{array}{cc}
1.3596 & 0.7988 \\
-2.4393 & -1.4775
\end{array}\right]
\end{aligned}
$$

\section{Conclusions}

This paper has addressed the stabilization of discrete linear repetitive processes with switched dynamics based on models for the cases when switching occurs in the pass-to-pass and along the pass directions respectively. In which context, note that the first of these does have immediate physical motivation in the form of metal rolling operations where a number of passes may be completed under one model of the process dynamics and then a subsequent number of passes are completed under a different model for the process dynamics. The results given here are also an important step towards analyzing more general switching in $2 \mathrm{D} / n D$ systems theory, such as extension to the more than two switched processes, encompassing the uncertain cases and also to switched differential processes (from pass to pass), which is the subject of on-going research.

As a final point, it should be noted that extending known results from $1 D$ systems with switched dynamics to the repetitive process $/ 2 D$ case is a non-trivial task, due to the repetitive 
nature of process dynamics and the structure of the boundary conditions at the start of each new pass. This means that the results obtained are much more complicated than the simple union of their $1 D$ systems counterparts but are still, as demonstrated in the examples given here, tractable and efficient. The boundary conditions assumed are the simplest possible in that they are independent of the previous pass profile. If, however, they are an explicit function of points along the previous pass then this alone can cause the process to be asymptotically unstable (and hence unstable along the pass). It should, however, be possible to extend the analysis given here to this more general case and this is the subject of ongoing research. Other current work is aimed at developing ways of undertaking reliable computations for very large dimensioned LMIs, with a view to limiting both the computational cost and the conservativeness associated with the methods used.

Acknowledgement This work is partially supported by Ministry of Scientific Research and Information Technology under the project 3 T11A 00826.

\section{References}

Agathoklis, P., \& Bruton, L. T. (1983). Practical-BIBO stability of n-dimensional discrete systems. Proceedings of The Institution of Electrical Engineers, 130(G), 236-242.

Amann, N., Owens, D. H., \& Rogers, E. (1998). Predictive optimal iterative learning control. International Journal of Control, 69(2), 203-226.

Benton, S. E. (2000). Analysis and control of linear repetitive processes. PhD Thesis, University of Southampton, UK.

D'Andrea, R., \& Dullerud, G. E. (2004). Distributed control of heterogeneous systems. IEEE Transactions on Automatic Control, 49(12), 2113-2128.

De Roover, D., Bosgra, O. H., \& Steinbuch, M. (2000). Internal-model-based design of repetitive and iterative learning controllers for linear multivariable systems. International Journal of Control, 73(10), 914-929.

Gałkowski, K., Rogers, E., Xu, S., Lam, J., \& Owens, D. H. (2002). LMIs—a fundamental tool in analysis and controller design for discrete linear repetitive processes. IEEE Transactions on Circuits and Systems I: Fundamental Theory and Applications, 49(6), 768-778.

Mehdi, D., Bachelier, O., \& Galkowski, K. (2004). State feedback stabilization of a class of discrete-time, linear, time variant systems. Proceedings of The International Symposium on the Mathematical Theory of Networks and Systems (MTNS 2004), CD Rom proceedings.

Moore K.L. (2000). A non-standard iterative learning control approach to tracking periodic signals in discrete-time nonlinear systems. International Journal of Control, 73(10), 955-967.

Norrlof M., \& Gunnarsson S. (2002). Time and frequency domain convergence properties in iterative learning control. International Journal of Control, 75(14), 1114-1126.

Peaucelle, D., Arzelier, D., Bachelier, O., \& Bernussou, J. (2000). A new robust D-stability condition for polytopic uncertainty. Systems and Control Letters, 40, 21-30.

Roberts, P. D. (2002). Two-dimensional analysis of an iterative nonlinear optimal control algorithm. IEEE Transactions on Circuits and Systems I: Fundamental Theory and Applications, 49(6), 872-878.

Rogers, E., \& Owens, D. H (1992). Stability Analysis for Linear Repetitive Processes. Springer-Verlag Lecture Notes in Control and Information Sciences Series, Vol. 175, Berlin.

Rogers, E., Galkowski, K., Gramacki, A., Gramacki, J., \& Owens, D. H (2002). Stability and controllability of a class of 2D linear systems with dynamic boundary conditions. IEEE Transactions on Circuits and Systems I: Fundamental Theory and Applications, 49(2), 181-195.

Rotariu I., Dijkstra B., \& Steinbuch M. (2004). Standard and lifted approaches of iterative learning control applied on a motion system. Proceedings of The International Symposium on the Mathematical Theory of Networks and Systems (MTNS 2004), CD Rom proceedings.

Smyth K. J. (1992). Computer aided analysis for linear repetitive processes. PhD Thesis, University of Strathclyde, Glasgow, UK. 


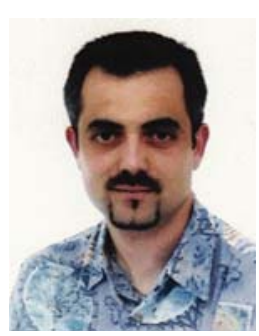

Olivier Bachelier was born in Chateauroux, France, in 1971. He received the "Diplome d'Ingenieur" (Engineer's Degree) and the "Diplome d'Etudes Approfondies" (Masters' Degree) with specialization in control from the "Institut National des Sciences Appliquées” (INSA, National Institute for Applied Sciences), Toulouse, France, in September 1994. From October 1995 to December 1998 he was a Ph.D. student at the "Laboratoire d'Analyse et d'Architecture des Systcmes" (LAAS, Laboratory of Analysis and Architecture of Systems) of the "Centre National de la Recherche Scientifique" (CNRS, National Center for Scientific Research) in Toulouse, France. He received the Ph.D. Degree from INSA in September 1998. Since 1999, he is "Maître de conférences" (Senior Lecturer) at the "Institut Universitaire de Technologie" (IUT, Academic Insititute of Technology, University of Poitiers) and researcher in the "Laboratoire d' Automatique et d'Informatique Industrielle" (LAII) of the " \'Ecole Sup \'erieure d'Ing \'enieurs de Poitiers" (ESIP, Superior Engineering School of Poitiers, University of Poitiers). His research interests are robust analysis of uncertain linear systems, robust D-stability, LMIs, and pole placement.

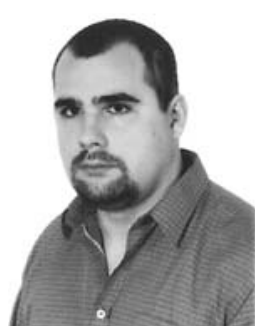

J. Bochniak received the M.Sc degree in control and computation engineering from the University of Zielona Gora, Zielona Gora, Poland, in 2002, where he is currently $\mathrm{Ph}$.D student. His research interests are in the area of multidimensional (nD) systems, repetitive processes, their theory and applications.

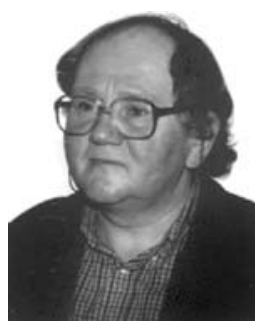

Krzysztof Galkowski received the M.S., Ph.D. and Habilitation degrees in electronics/automatic control from Technical University of Wroclaw, Poland in 1972, 1977 and 1994 respectively. In October 1996 he joined the Technical University of Zielona Góra (now the University of Zielona Góra), Poland where he holds the Professor position. He has been awarded by the Visiting Professor position in the University of Southampton, UK. He is an awardee of the Siemens-Poland 2004 award. Also, he was the Gerhard Mercator Guest Professor in Wuppertal, Germany during the academic year 2004-2005. His research interests include multivariable (nD) systems, repetitve processes-theory and applications, control and related numerical and symbolic algebra methods. He is an author of the monograph: Galkowski K., State-space Realizations of Linear 2-D Systems with Extensions to the General nD ( $\mathrm{n}>2$ ) Case, Springer Verlag, LNCIS 2001. Co-editor of Galkowski K., Wood J., Eds., Multidimensional Signals, Circuits and Systems, Taylor \& Francis, 2001 and around 100 research texts published in the leading journals and proceedings of international conferences.

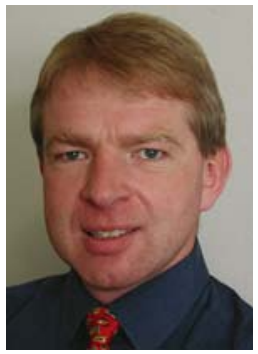

Anton Kummert received the Dipl.-Ing. (FH) degree in electrical engineering from the Fachhochschule Coburg, Coburg, Germany, in 1982, and the Dipl.-Ing. and Dr.-Ing. degrees from the Ruhr-Universität Bochum, Bochum, Germany, in 1985 and 1988 respectively. From 1985 to 1991, he was a Research Assistant at the Department of Electrical Engineering of the Ruhr-Universität Bochum. From 1991 to 1995, he was employed by STN Atlas Elektronik, Bremen, Germany. Since 1995 he has been a professor for Communication Theory at University of Wuppertal, Wuppertal, Germany. Dr. Kummert is a recipient of the "HeinrichKost-Preis" (1989) and of the "Akademie-Preis 1990" of the Rheinisch-Westfälische Akademie der Wissenschaften (1990). He is a member of VDE, ITG and Senior member of IEEE. He is and has been member of the editorial board of several international journals, organizer and chairman of numerous sessions at major conferences, and one of the General Chairs and organizers of the 4th Int. Workshop on Multidimensional (ND) Systems. His current research interests comprise multidimensional systems and circuit theory, image processing and generation, wireless ad hoc networks, and tomographic reconstruction methods. 


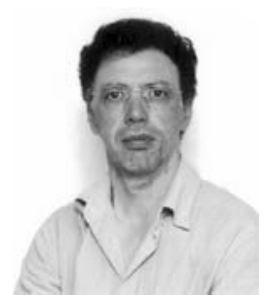

Driss MEHDI was born in Oujda, Morocco in 1955. He recieved an engineer degree from Mohammadia Engineering school, Rabat, Morocco in 1979 and the Ph.D in Automatic control from Nancy University in 1986. He was Senior Lecturer from 1988 to 1992 in Louis Pasteur University in Strasbourg and from 1992 he was appointed as a Professor in the University of Poitiers. His main interest are Automatic control, Robust control, LMIs and Delay systems.

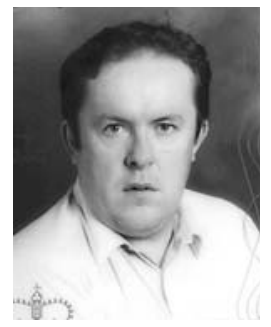

Eric Rogers was born in 1956 near Dungannon in Northern Ireland. He read Mechanical Engineering as an undergraduate in The Queen's University of Belfast UK and was awarded his PhD degree by The University of Sheffield UK for a Thesis in the area of Multidimensional Systems Theory. He has been with the University of Southampton UK since 1990 where he is currently Professor of Control Systems Theory and Design in The School of Electronics and Computer Science. Prior to moving to Southampton, he held lectureship posts in The Queen's University of Belfast UK (1984-1987) and the University of Strathclyde UK (1988-1990). His current major research interests include multidimensional systems theory and applications, with particular emphasis on Behavioral Systems Theory Approaches and systems with Repetitive Dynamics, Iterative Learning Control, Flow Control, and Active Control of Microvibrations. He is currently the editor of The International Journal of Control, an associate editor of Multidimensional Systems and Signal Processing, and joint editor of The Taylor and Francis research book series on Systems and Control. In addition, he has served extensively on IEEE, IFAC and IEE technical committees and acted as a consultant to numerous companies and government agencies in UK and abroad. 